\title{
The resolvent cocycle in twisted cyclic cohomology and a local index formula for the Podleś sphere
}

\author{
Adam Rennie and Roger Senior
}

\begin{abstract}
We continue the investigation of twisted homology theories in the context of dimension drop phenomena. This work unifies previous equivariant index calculations in twisted cyclic cohomology. We do this by proving the existence of the resolvent cocycle, a finitely summable analogue of the JLO cocycle, under weaker smoothness hypotheses and in the more general setting of 'modular' spectral triples. As an application we show that using our twisted resolvent cocycle, we can obtain a local index formula for the Podleś sphere. The resulting twisted cyclic cocycle has non-vanishing Hochschild class which is in dimension 2.
\end{abstract}

Mathematics Subject Classification (2010). 19K35, 19K56, 46L80, 46L87.

Keywords. Spectral triple, cyclic cohomology, Kasparov theory, q-deformations, Podleś sphere.

\section{Contents}

1 Introduction . . . . . . . . . . . . . . . . . . 2

2 Modular spectral triples and equivariant K-theory . . . . . . . . . . . . . 3

2.1 Modular spectral triples ...................... 3

2.2 Equivariant KK-theory and modular spectral triples . . . . . . . . . . . 6

3 The resolvent index formula in twisted cyclic cohomology . . . . . . . . . . 8

3.1 Weakly $\mathrm{Q} C^{\infty}$ modular spectral triples . . . . . . . . . . . 8

3.2 Existence of the resolvent cocycle for weakly $\mathrm{Q} C^{\infty}$ modular spectral triples . 15

3.3 The resolvent index formula for modular spectral triples . . . . . . . . . . 20

4 The local index formula for the Podleś sphere . . . . . . . . . . . . . . . . . . . . . . . . . .

4.1 The modular spectral triple for the Podleś sphere . . . . . . . . . . . . . 26

4.2 The residue cocycle for the Podleś sphere . . . . . . . . . . . . . . . 30

4.3 Some equivariant projections and their Chern characters . . . . . . . . . . 35

4.4 The index pairing . . . . . . . . . . . . . . . . . . . . . . . . . . . . . . . . .

References . . . . . . . . . . . . . . . . . . 40 


\section{Introduction}

This paper proves a residue index formula in noncommutative geometry for 'modular spectral triples', which are analogues of spectral triples with twisted traces. This is the appropriate setting for examples arising from $q$-deformations which typically experience 'dimension drop' in homology, [H], [HK], [NT], [SW], [W]. The main results are as follows.

1) We show that for finitely summable modular spectral triples the resolvent cocycle exists, is continuous and is an index cocycle under weaker smoothness conditions than have previously been used. In particular we do not need the pseudodifferential calculus to establish these facts, so that we are free to replace the usual pseudodifferential calculus by other schemes later, in order to obtain local index formulae.

2) We show that modular spectral triples have a well-defined pairing with equivariant K-theory. In the finitely summable and weakly smooth case we show that this pairing can be computed using the resolvent cocycle, which defines a twisted cyclic cocycle.

3) We apply the results of 1) and 2) to prove a local index formula for the Podles sphere in twisted cyclic cohomology. This index formula puts the results of several authors into a common framework, $[\mathrm{H}],[\mathrm{KW}],[\mathrm{W}]$. In particular, the twisted Hochschild class of our residue cocycle is an explicit constant multiple of the fundamental Hochschild cocycle for the Podleś sphere, [H], [KW], and our explicit index pairings can be compared to those in [W].

The computations in 3) are similar to what was done in [NT], however they used the twisting by the modular automorphism, rather than the inverse of the modular automorphism. While the summability is the same in both cases, the twisted Hochschild homology for the modular automorphism is trivial in dimension 2, while the inverse of the modular automorphism avoids the dimension drop. Thus the cocycle obtained in $[\mathrm{NT}]$ is cohomologous to a 0 -cocycle, while ours is not. We also note that in [NT] the starting point was the JLO cocycle in entire cyclic cohomology rather than the resolvent cocycle.

The exposition is as follows. In Section 2 we introduce the basic definitions for modular spectral triples, including smoothness and summability. We then show that a modular spectral triple defines an equivariant KK-class, and so gives us a wellposed K-theory valued index problem. The remainder of Section 2 demonstrates that together with a representative of an equivariant K-theory class, we obtain a well-posed numerical index problem. The aim of Section 3 is then to show that these notions are compatible.

We address the existence, continuity and index properties of the resolvent cocycle in Section 3. We begin by looking at our weak smoothness condition, and proving some basic results that follow from this assumption. Then we prove the existence and continuity of the resolvent cocycle, which originated in [CPRS2], and show that it computes the numerical index. Finally we show, using results from [KNR], that this numerical index is compatible with the K-theory valued index in a precise way. 
In Section 4 we show that the spectral triple introduced by [DS] defines a 2dimensional modular spectral triple, which is weakly smooth in our sense. Numerous results of [KW], [NT], [SW], [W] are incorporated into this statement. We employ Neshveyev and Tuset's modification of the pseudodifferential calculus to obtain a version of the local index formula for the Podleś sphere. Thus we see that with a suitable pseudodifferential calculus, our resolvent index formula can be extended to a full local index formula as in [CPRS2], [CM], [Hig]. We conclude by computing some explicit index pairings, and as a corollary see that the degree two term in the residue index cocycle is not a coboundary.

Acknowledgements. It is a pleasure to acknowledge the assistance of our colleagues Alan Carey, Ulrich Krähmer and Joe Várilly. We would also like to thank the referee for numerous comments which have improved the exposition. Both authors were supported by the Australian Research Council.

\section{Modular spectral triples and equivariant K-theory}

We begin this section by defining modular spectral triples, a generalisation of semifinite spectral triples, [BeF], [CP2], [CPRS2], allowing for twisted traces (weights) in place of traces. We then consider the index pairings defined by modular spectral triples.

The strategy to study index pairings is almost the same as in [CPRS2], [CPRS3]. Given a representative of an equivariant K-theory class for an algebra $\mathcal{A}$, we show that a modular spectral triple over $\mathcal{A}$ allows us to formulate a well-defined (semi-finite) index problem. By following the strategy of [CPRS2], [CPRS3], we find that the index can be computed by pairing a cocycle with the Chern character of the K-theory class.

2.1. Modular spectral triples. Let $\mathcal{N}$ be a semi-finite von Neumann algebra acting on a Hilbert space $\mathscr{H}$, and fix a faithful normal semi-finite weight $\phi$. We denote the modular automorphism group of $\phi$ by $\sigma_{t}^{\phi}$. Then as $\phi$ is $\sigma_{t}^{\phi}$ invariant, we see that for all $T \in \operatorname{dom} \phi \subset \mathcal{N}$ and $t \in \mathbb{R}$,

$$
\phi(T)=\phi\left(\sigma_{t}^{\phi}(T)\right) .
$$

Suppose further that the modular group $\sigma_{t}^{\phi}$, which is inner since $\mathcal{N}$ is semi-finite, is periodic, and let $\alpha$ be the (least) period of $\sigma_{t}^{\phi}$. Then

$$
\phi(T)=\frac{1}{\alpha} \int_{0}^{\alpha} \phi\left(\sigma_{t}^{\phi}(T)\right) d t=\phi\left(\frac{1}{\alpha} \int_{0}^{\alpha} \sigma_{t}^{\phi}(T) d t\right)=:(\phi \circ \Psi)(T),
$$

where $\Psi: \mathcal{N} \rightarrow \mathcal{M}:=\mathcal{N}^{\sigma^{\phi}}$ is the expectation onto the fixed point algebra $\mathcal{M}$ defined by the integral. Then the restriction of $\phi$ to $\mathcal{M}$ is a faithful normal trace. 
The restriction of $\phi$ to $\mathcal{M}$ is also semi-finite if and only if $\phi$ is strictly semi-finite, meaning that $\phi$ is the sum of normal positive linear functionals whose supports are mutually orthogonal, [T], p. 105. In everything that follows, we suppose that $\phi$ is strictly semi-finite.

Given a faithful normal semi-finite trace $\tau$ on a von Neumann algebra $\mathcal{N}$, we define the ideal of $\tau$-compact operators $\mathcal{K}(\mathcal{N}, \tau)$ to be the norm closure of the ideal generated by the projections $p$ with finite trace, $\tau(p)<\infty$.

Definition 2.1. Let $\mathcal{N}$ be a semi-finite von Neumann algebra acting on a Hilbert space $\mathcal{H}$, and fix a faithful normal strictly semi-finite weight $\phi$. Suppose further that the modular group $\sigma_{t}^{\phi}$ is periodic. Then we say that $(\mathcal{A}, \mathcal{H}, \mathscr{D})$ is a unital modular spectral triple with respect to $(\mathcal{N}, \phi)$ if

(1) $\mathcal{A}$ is a separable unital $*$-subalgebra of $\mathcal{N}$ with norm closure $A$;

(2) $\mathcal{A}$ is invariant under $\sigma^{\phi}, \mathcal{A}$ consists of analytic vectors for $\sigma^{\phi}$, and $\left.\sigma^{\phi}\right|_{A}$ is a strongly continuous action;

(3) $\mathcal{D}$ is a self-adjoint operator affiliated to the fixed point algebra $\mathcal{M}:=\mathcal{N}^{\sigma^{\phi}}$;

(4) $[\mathcal{D}, a]$ extends to a bounded operator in $\mathcal{N}$ for all $a \in \mathcal{A}$;

(5) $\left(1+\mathscr{D}^{2}\right)^{-\frac{1}{2}} \in \mathcal{K}\left(\mathcal{M},\left.\phi\right|_{\mathcal{M}}\right)$.

The triple is even if there exists $\gamma=\gamma^{*} \in \mathcal{M}$ with $\gamma^{2}=1, \gamma a=a \gamma$ for all $a \in \mathcal{A}$ and $\gamma \mathscr{D}+\mathscr{D} \gamma=0$. Otherwise the triple is odd.

We say that the triple is finitely summable with spectral dimension $p \geq 1$ if $p$ is the least number such that

$$
\phi\left(\left(1+D^{2}\right)^{-\frac{s}{2}}\right)<\infty \text { for all } \operatorname{Re}(s)>p .
$$

Just as for ordinary spectral triples, there is a notion of smoothness and pseudodifferential operators for $\mathrm{Q} C^{\infty}$ modular spectral triples, just as in [CPRS2], [CM], which we recall here.

Definition 2.2. A modular spectral triple $(\mathcal{A}, \mathscr{H}, \mathcal{D})$ relative to $(\mathcal{N}, \phi)$ is $\mathrm{Q} C^{k}$ for $k \geq 1$ (Q for quantum) if for all $a \in \mathcal{A}$ the operators $a$ and [D,a] are in the domain of $\delta_{1}^{k}$, where $\delta_{1}(T)=\left[\left(1+\mathcal{D}^{2}\right)^{\frac{1}{2}}, T\right]$ is the partial derivation on $\mathcal{N}$ defined by $\left(1+\mathscr{D}^{2}\right)^{\frac{1}{2}}$. We say that $(\mathcal{A}, \mathcal{H}, \mathscr{D})$ is $\mathrm{Q} C^{\infty}$ if it is $\mathrm{Q} C^{k}$ for all $k \geq 1$. Equivalently, [CPRS2], Proposition 6.5, and [CM], Lemma B.2, $(\mathcal{A}, \mathscr{H}, \mathcal{D})$ is $\mathrm{Q} C^{\infty}$ if for all $a \in \mathcal{A}$ we have $a,[\mathcal{D}, a] \in \bigcap_{k, l \geq 0} \operatorname{dom} L_{1}^{k} \circ R_{1}^{l}$, where $L, R$ are defined by

$$
L(T)=\left(1+\mathscr{D}^{2}\right)^{-\frac{1}{2}}\left[\mathscr{D}^{2}, T\right]=:\left(1+\mathscr{D}^{2}\right)^{-\frac{1}{2}} T^{(1)}
$$

and

$$
R(T)=\left[\mathscr{D}^{2}, T\right]\left(1+\mathscr{D}^{2}\right)^{-\frac{1}{2}}=: T^{(1)}\left(1+\mathscr{D}^{2}\right)^{-\frac{1}{2}} .
$$

Here we have introduced the notation $T^{(1)}:=\left[\mathscr{D}^{2}, T\right]$, and for $n>1$ we recursively set $T^{(n)}:=\left[\mathscr{D}^{2}, T^{(n-1)}\right]$. 
Definition 2.3. Let $(\mathcal{A}, \mathscr{H}, \mathcal{D})$ be a modular spectral triple relative to $(\mathcal{N}, \phi)$. For $r \in \mathbb{R}$ put

$$
\mathrm{OP}^{r}=\left(1+\mathscr{D}^{2}\right)^{\frac{r}{2}}\left(\bigcap_{n \geq 0} \operatorname{dom} \delta_{1}^{n}\right) .
$$

If $T \in \mathrm{OP}^{r}$ we say that $T$ is a pseudodifferential operator and that the order of $T$ is (at most) $r$. The definition is actually symmetric, since for $r$ an integer (at least) we have by [CPRS2], Lemma 6.2,

$$
\begin{aligned}
\mathrm{OP}^{r} & =\left(1+\mathcal{D}^{2}\right)^{\frac{r}{2}}\left(\bigcap \operatorname{dom} \delta_{1}^{n}\right) \\
& =\left(1+\mathcal{D}^{2}\right)^{\frac{r}{2}}\left(\bigcap \operatorname{dom} \delta_{1}^{n}\right)\left(1+\mathscr{D}^{2}\right)^{-\frac{r}{2}}\left(1+\mathscr{D}^{2}\right)^{\frac{r}{2}} \\
& \subseteq\left(\bigcap \operatorname{dom} \delta_{1}^{n}\right)\left(1+\mathscr{D}^{2}\right)^{\frac{r}{2}} .
\end{aligned}
$$

From this we easily see that $\mathrm{OP}^{r} \cdot \mathrm{OP}^{s} \subseteq \mathrm{OP}^{r+s}$. Finally, we note that if $b \in \mathrm{OP}^{r}$ for $r \geq 0$, then since $b=\left(1+\mathscr{D}^{2}\right)^{\frac{r}{2}} a$ for some $a \in \mathrm{OP}^{0}$, we get $\left[\left(1+\mathscr{D}^{2}\right)^{\frac{1}{2}}, b\right]=$ $\left(1+\mathscr{D}^{2}\right)^{\frac{r}{2}}\left[\left(1+\mathscr{D}^{2}\right)^{\frac{1}{2}}, a\right]=\left(1+\mathscr{D}^{2}\right)^{\frac{r}{2}} \delta_{1}(a)$, so $\left[\left(1+\mathscr{D}^{2}\right)^{\frac{1}{2}}, b\right] \in \mathrm{OP}^{r}$.

Remarks. 1) An operator $T \in \mathrm{OP}^{r}$ if and only if $\left(1+\mathscr{D}^{2}\right)^{-\frac{r}{2}} T \in \bigcap_{n \geq 0} \operatorname{dom} \delta_{1}^{n}$. Observe that operators of order at most zero are bounded.

2) We will need a weaker notion of smoothness, introduced in Section 3, for modular spectral triples, as Definition 2.2 is not satisfied for our main example, the Podleś sphere.

Example. A semi-finite spectral triple is a modular spectral triple with $\phi$ a semi-finite normal trace (and so $\mathcal{M}=\mathcal{N}$ ).

Example. Given a circle action on a unital $\mathrm{C}^{*}$-algebra $A$, every state on $A$ which is KMS for this circle action gives rise to a modular spectral triple of dimension 1. Explicit examples are the Cuntz algebra with its usual gauge action, [CPR2], and the quantum group $\mathrm{SU}_{q}(2)$ with its Haar state, [CRT]. All these examples are $\mathrm{Q} C{ }^{\infty}$ (or regular or smooth) when we use the algebra of analytic vectors $\mathcal{A} \subset A$ for the circle action. More examples arising from a topological version of the group-measure space construction are presented in [CPPR]. In all these examples the von Neumann algebra is a proper subalgebra of $\mathcal{B}(\mathscr{H})$, where $\mathscr{H}$ is the Hilbert space of the modular spectral triple.

Example. The only other unital example (known to the authors) is the Podles sphere, which provides an example of a modular spectral triple of dimension 2 . This was first presented in [DS], and has been studied in numerous subsequent works by various authors. The paper [W] provides a good summary. This example is not $\mathrm{Q} C^{\infty}$, but a replacement for the pseudodifferential calculus was developed in [NT]. This example is our main motivation for weakening the $\mathrm{Q} C^{\infty}$ condition, and this example will be presented in detail in Section 4. 
Nonunital examples. We have chosen to work in the unital case for simplicity, but there are nonunital examples, [CNNR], [CMR]. However, to simplify the discussion of the local index formula, we will restrict to the unital case. To handle the nonunital case in general, we would need to modify the definition of modular spectral triple in order to utilise (analogues of) the results of [CGRS2], where the local index formula is proved in the nonunital case.

2.2. Equivariant KK-theory and modular spectral triples. An odd modular spectral triple $(\mathcal{A}, \mathcal{H}, \mathcal{D})$ with respect to $(\mathcal{N}, \phi)$ defines an equivariant Kasparov module, and so a class $[D \mathcal{D}] \in \mathrm{KK}^{1, \mathbb{T}}\left(A, \mathcal{K}_{\mathcal{N}}\right)$, where we recall that $A=\overline{\mathcal{A}}$. The construction of the Kasparov module associated to a modular spectral triple begins with the definition of a suitable ideal. We will deal explicitly with the odd case here, just stating the analogous results in the even case.

Definition 2.4. Given a modular spectral triple $(\mathcal{A}, \mathscr{H}, \mathcal{D}, \mathcal{N}, \phi)$, let

$$
J_{\phi}:=\left\{S k T \mid S, T \in \mathcal{N}, k \in \mathcal{K}\left(\mathcal{M},\left.\phi\right|_{\mathcal{M}}\right)\right\}
$$

denote the norm closed two-sided ideal in $\mathcal{N}$ generated by $\mathcal{K}\left(\mathcal{M},\left.\phi\right|_{\mathcal{M}}\right)$.

The ideal $J_{\phi}$ is a right Hilbert module over itself, and $\mathcal{A}$ acts on the left of $J_{\phi}$ by multiplication. The axioms of a modular spectral triple imply that $\left(1+\mathscr{D}^{2}\right)^{-\frac{1}{2}} \in J_{\phi}$. With a little effort we can show, as in [KNR], Theorem 4.1, that the pair $\left(J_{\phi}, \mathcal{D}(1+\right.$ $\left.\mathscr{D}^{2}\right)^{-\frac{1}{2}}$ ) is a Kasparov module, except that the module $J_{\phi}$ may not be countably generated.

To deal with this problem, we recall the following construction from [KNR], Theorem 5.3.

Definition 2.5. Let $(\mathcal{A}, \mathscr{H}, \mathcal{D}, \mathcal{N}, \phi)$ be a modular spectral triple, where we recall that $\mathcal{A}$ is separable. Write $F_{\mathscr{D}}:=\mathscr{D}\left(1+\mathscr{D}^{2}\right)^{-\frac{1}{2}}$ and let $B_{\phi}$ be the smallest $\mathrm{C}^{*}$ algebra in $\mathcal{N}$ containing the elements

$$
F_{\mathscr{D}}\left[F_{\mathscr{D}}, a\right], \quad b\left[F_{\mathscr{D}}, a\right], \quad F_{\mathscr{D}} b\left[F_{\mathscr{D}}, a\right], \quad a \varphi(\mathscr{D})
$$

for all $a, b \in \mathcal{A}$ and $\varphi \in C_{0}(\mathbb{R})$. Then $B_{\phi}$ is separable, and so $\sigma$-unital, and contained in $J_{\phi}$.

Proposition 2.6. A modular spectral triple $(\mathcal{A}, \mathcal{H}, \mathcal{D}, \mathcal{N}, \phi)$ defines an equivariant $K K$-theory class $[\mathscr{D}]=\left[B_{\phi}, F_{\mathscr{D}}\right] \in \mathrm{KK}^{1, \mathbb{T}}\left(A, B_{\phi}\right)$, where $F_{\mathscr{D}}:=\mathscr{D}\left(1+\mathscr{D}^{2}\right)^{-\frac{1}{2}}$.

Proof. A modular spectral triple is automatically a von Neumann spectral triple with respect to $J_{\phi}$ in the sense of [KNR]. Then [KNR], Theorem 5.3, shows that $B_{\phi}$ is a countably generated right $\mathrm{C}^{*} B_{\phi}$-module, and that the pair $\left(B_{\phi}, F_{\mathscr{D}}\right)$ is a Kasparov module. The equivariance is immediate. 
Having obtained an equivariant Kasparov module, and so a KK-class, the Kasparov product defines a $K_{0}^{\mathbb{T}}\left(B_{\phi}\right)$-valued index pairing between a modular spectral triple and equivariant K-theory. That is,

$$
K_{1}^{\mathbb{T}}(A) \times \mathrm{KK}^{1, \mathbb{T}}\left(A, B_{\phi}\right) \rightarrow K_{0}^{\mathbb{T}}\left(B_{\phi}\right) .
$$

See [B], Theorem 18.4.4, for example. We now seek an analytic formula to compute this index, and in Section 3 we obtain such a formula, the resolvent index formula. The first step is the construction of a semi-finite spectral triple which encodes the index pairing between a modular spectral triple and an equivariant K-theory class. This is necessary to obtain a well-defined numerical index problem. We now describe this procedure.

Given a modular spectral triple $(\mathcal{A}, \mathscr{H}, \mathcal{D}, \mathcal{N}, \phi)$ and a class $[u] \in K_{1}^{\mathbb{T}}(A)$, there is a unitary $u \in M_{n}(\mathcal{A})$ and a representation $V: \mathbb{T} \rightarrow M_{n}(\mathbb{C})$ such that $u$ is $\sigma^{\phi} \otimes \operatorname{Ad} V$ invariant, [B], [CNNR]. In particular, if $n=1$ then $u$ is $\sigma^{\phi}$ invariant.

We can diagonalise the representation $V_{t}=\bigoplus_{j=1}^{n} \lambda_{j}^{i t}, \lambda_{j} \in[1, \infty)$, and in this basis it is clear that

(1) $u_{i j}$ transforms under Ad $V_{t}$ by $\lambda_{i}^{i t} \lambda_{j}^{-i t}$;

(2) $u_{i j}$ transforms under $\sigma_{t}^{\phi}$ by $\lambda_{i}^{-i t} \lambda_{j}^{i t}$;

(3) $V_{t}$ extends to an action of $\mathbb{C}$ which is not a $*$-action but satisfies $V_{z}^{*}=V_{-\bar{z}}$.

We define a positive functional $G: M_{n}(\mathbb{C}) \rightarrow \mathbb{C}$ by setting

$$
G(T)=\operatorname{Tr}\left(V_{-i} T\right), \quad T \in M_{n}(\mathbb{C}) .
$$

Then $G$ is a $\mathrm{KMS}_{1}$ functional on $M_{n}(\mathbb{C}),[\mathrm{BR}]$, for the action Ad $V$, but is not a state as it is not normalised.

Now consider the fixed point algebra $\mathcal{M}_{n}=\left(M_{n}(\mathcal{N})\right)^{\sigma^{\phi} \otimes \operatorname{Ad} V}$, which is the centralizer of the weight $\phi \otimes G,[\mathrm{~T}]$, Proposition 4.3. Then $\phi \otimes G$ restricts to a faithful normal semi-finite trace on $\mathcal{M}$ and moreover $u \in \mathcal{M}_{n}$. The latter statement follows from the definition of $u$. The former follows since the strict semi-finiteness of $\phi$ implies the strict semi-finiteness of $\phi \otimes G$.

Lemma 2.7. Let $(\mathcal{A}, \mathscr{H}, \mathcal{D}, \mathcal{N}, \phi)$ be a modular spectral triple which is finitely summable and $u \in M_{n}(\mathcal{A})$ a $\sigma^{\phi}$ equivariant unitary, with associated representation $V: \mathbb{T} \rightarrow M_{n}(\mathbb{C})$. Then

$$
\left(C^{\infty}(u), \mathscr{H} \otimes \mathbb{C}^{n}, \mathcal{D} \otimes \operatorname{Id}_{n}, \mathcal{M}_{n}, \phi \otimes G\right)
$$

is a finitely summable semi-finite spectral triple. Here $C^{\infty}(u)$ is the algebra of all $f(u) \in C^{*}(u)$ with $f$ a $C^{\infty}$ function on the spectrum of $u$. Let $B_{\phi \otimes G} \subseteq$ $\mathcal{K}\left(\mathcal{M}_{n}, \phi \otimes G\right)$ be defined as in Definition 2.5. Then this semi-finite spectral triple defines a Kasparov class in $\mathrm{KK}^{1, \mathbb{T}}\left(C^{*}(u), B_{\phi \otimes G}\right)$. 
Proof. The statement that we obtain a semi-finite spectral triple follows from the construction, and that we get a Kasparov module follows from Proposition 2.6.

Thus given $[u, V] \in K_{1}^{\mathbb{T}}(\mathcal{A})$, we apply [KNR], Theorem 6.9, to compute the spectral flow, [Ph]. Let $i: B_{\phi \otimes G} \hookrightarrow \mathcal{K}\left(\mathcal{M}_{n}, \phi \otimes G\right)$ be the inclusion, and let $i_{*}: K_{0}\left(B_{\phi \otimes G}\right) \rightarrow K_{0}\left(\mathcal{K}\left(\mathcal{M}_{n}, \phi \otimes G\right)\right)$. Then [KNR], Theorem 6.9, allows us to compute the spectral flow as

$$
s f_{\phi \otimes G}\left(\mathscr{D} \otimes \operatorname{Id}_{n}, u\left(\mathscr{D} \otimes \operatorname{Id}_{n}\right) u^{*}\right)=(\phi \otimes G)_{*}\left(i_{*}\left([u] \otimes_{C^{*}(u)}\left[\mathscr{D} \otimes \operatorname{Id}_{n}\right]\right)\right) .
$$

At this point, we have obtained an index problem which a priori depends on the representative $u$ of the equivariant K-theory class (through the use of $C^{*}(u)$ ). To show that we do indeed have a well-defined pairing with $K_{1}^{\mathbb{T}}(\mathcal{A})$, we will show, via the resolvent index formula, that the index can be computed in terms of the Chern character of $u$, which is independent of the chosen representative of the class $[u]$. Finally, we show that the original index pairing between a modular spectral triple and equivariant K-theory can be described by the spectral flow above.

\section{The resolvent index formula in twisted cyclic cohomology}

In this section we express the spectral flow from eq. (2.1) in terms of the pairing between a twisted cyclic cocycle dependent only on the modular spectral triple and the Chern character of the equivariant unitary. In order to achieve this without invoking the $\mathrm{Q} C^{\infty}$ property, we make a technical improvement on the work of [CPRS2] by using a weaker smoothness condition. This is necessary for our application, as the Podleś sphere modular spectral triple is not $\mathrm{Q} C^{\infty}$.

3.1. Weakly $\mathrm{Q} C^{\infty}$ modular spectral triples. We weaken the $\mathrm{Q} C^{\infty}$ condition with the aim of justifying a resolvent expansion, used in the proof of our index formulae, without recourse to the pseudodifferential calculus. There are two basic reasons for doing this.

The first is that the example of the Podleś sphere shows that we do not always have the $\mathrm{Q} C^{\infty}$ property for modular spectral triples.

The second reason is that, conceptually, the use of the pseudodifferential calculus to prove existence and continuity of the resolvent cocycle is overkill, requiring us to invoke much more smoothness than is necessary for the statement of existence and continuity.

Definition 3.1. Let $(\mathcal{A}, \mathscr{H}, \mathcal{D})$ be a modular spectral triple relative to $(\mathcal{N}, \phi)$. For $T \in \mathcal{N}$ mapping the domain of $\mathscr{D}^{2}$ to itself, define

$$
\begin{aligned}
& W L(T):=\left(1+\mathscr{D}^{2}\right)^{-1}\left[\mathscr{D}^{2}, T\right]=\left(1+\mathscr{D}^{2}\right)^{-1} T\left(1+\mathscr{D}^{2}\right)-T, \\
& W R(T):=\left[\mathscr{D}^{2}, T\right]\left(1+\mathscr{D}^{2}\right)^{-1}=\left(1+\mathcal{D}^{2}\right) T\left(1+\mathscr{D}^{2}\right)^{-1}-T .
\end{aligned}
$$


We say that $(\mathcal{A}, \mathscr{H}, \mathscr{D})$ is weakly $\mathrm{Q} C^{\infty}$ if

$$
\mathcal{A} \subset \mathrm{OP}^{0} \subset \mathcal{N} \quad \text { and }[\mathcal{D}, \mathcal{A}] \subset \mathrm{w}^{-} \mathrm{OP}^{0}:=\bigcap_{k, l \geq 0} \operatorname{dom}(W L)^{k}(W R)^{l} \subset \mathcal{N} \text {. }
$$

The analogous definition of weak $\mathrm{Q} C^{k}$ is awkward, since in Definition 2.2, Q $C^{k}$ is defined in terms of commutators with $|\mathscr{D}|$ or $\left(1+\mathscr{D}^{2}\right)^{\frac{1}{2}}$. We will leave aside these questions and just work with weak $\mathrm{Q} C^{\infty}$. Also, $\mathrm{Q} C^{\infty}$ implies weak $\mathrm{Q} C^{\infty}$ by the boundedness of $\left(1+\mathscr{D}^{2}\right)^{-\frac{1}{2}}$.

While we do not have a pseudodifferential calculus for a weakly $\mathrm{Q} C^{\infty}$ modular spectral triple $(\mathcal{A}, \mathcal{H}, \mathcal{D})$, we may consider the weak pseudodifferential operators of order $s \in \mathbb{R}$ given by

$$
\mathrm{w}-\mathrm{OP}^{s}:=\left(1+\mathcal{D}^{2}\right)^{\frac{s}{2}}\left(\bigcap_{k, l} \operatorname{dom} W L^{k} \circ W R^{l}\right) .
$$

This definition is symmetric, in the sense that

$$
\mathrm{w}-\mathrm{OP}^{s}=\left(\bigcap_{k, l} \operatorname{dom} W L^{k} \circ W R^{l}\right)\left(1+\mathcal{D}^{2}\right)^{\frac{s}{2}}
$$

since for all $s \in \mathbb{R}, \mathrm{w}-\mathrm{OP}^{s}$ is preserved by $T \mapsto\left(1+\mathscr{D}^{2}\right)^{ \pm s} T\left(1+\mathscr{D}^{2}\right)^{\mp s}$, by Lemma 3.2 below. Observe also that we have $\mathrm{OP}^{s} \subset$ w-OP ${ }^{s}$.

It follows from the definitions that if $(\mathcal{A}, \mathscr{H}, \mathcal{D})$ is a weakly $\mathrm{Q} C{ }^{\infty}$ modular spectral triple and $u \in M_{n}(\mathcal{A})$ is an equivariant unitary, then the associated semifinite spectral triple $\left(C^{\infty}(u), \mathscr{H} \otimes \mathbb{C}^{n}, \mathscr{D} \otimes \mathrm{Id}_{n}, \mathcal{M}_{n}, \phi \otimes G\right)$ is also weakly $\mathrm{Q} C^{\infty}$.

The next few lemmas record some basic properties of the maps $W L$ and $W R$.

Lemma 3.2. Let $\mathscr{D}: \operatorname{dom} \mathscr{D} \subset \mathscr{H} \rightarrow \mathscr{H}$ be an unbounded self-adjoint operator. Then $T \in \mathscr{B}(\mathscr{H})$ belongs to

$$
\bigcap_{k, l \geq 0} \operatorname{dom}(W L)^{k}(W R)^{l}
$$

if and only if $\left(1+\mathscr{D}^{2}\right)^{\frac{s}{2}} T\left(1+\mathscr{D}^{2}\right)^{-\frac{s}{2}}$ extends to a bounded operator for all $s \in \mathbb{R}$.

Proof. It follows from Definition 3.1 that $T \in \bigcap_{k, l \geq 0} \operatorname{dom}(W L)^{k}(W R)^{l}$ if and only if $\left(1+\mathscr{D}^{2}\right)^{k} T\left(1+\mathscr{D}^{2}\right)^{-k}$ is a bounded operator for all $k \in \mathbb{Z}$. It is also immediate that if $\left(1+\mathscr{D}^{2}\right)^{\frac{s}{2}} T\left(1+\mathscr{D}^{2}\right)^{-\frac{s}{2}}$ is bounded for all $s \in \mathbb{R}$, then $T \in \bigcap_{k, l \geq 0} \operatorname{dom}(W L)^{k}(W R)^{l}$. So let $0<s<1$, and recall that

$$
\left(1+\mathscr{D}^{2}\right)^{-s}=\frac{\sin (s \pi)}{\pi} \int_{0}^{\infty} \lambda^{-s}\left(1+\lambda+\mathscr{D}^{2}\right)^{-1} d \lambda .
$$


Then for $T \in \bigcap_{k, l \geq 0} \operatorname{dom}(W L)^{k}(W R)^{l}$ we have

$$
\begin{aligned}
\left(1+\mathscr{D}^{2}\right)^{s} T\left(1+\mathscr{D}^{2}\right)^{-s} & \\
= & \left(1+\mathscr{D}^{2}\right)^{s} T \frac{\sin (s \pi)}{\pi} \int_{0}^{\infty} \lambda^{-s}\left(1+\lambda+\mathscr{D}^{2}\right)^{-1} d \lambda \\
= & \left(1+\mathscr{D}^{2}\right)^{s} \frac{\sin (s \pi)}{\pi} \int_{0}^{\infty} \lambda^{-s}\left(\left(1+\lambda+\mathscr{D}^{2}\right)^{-1} T\right. \\
& \left.\quad+\left(1+\lambda+\mathscr{D}^{2}\right)^{-1}\left[\mathscr{D}^{2}, T\right]\left(1+\lambda+\mathscr{D}^{2}\right)^{-1}\right) d \lambda \\
= & \left(1+\mathscr{D}^{2}\right)^{s} \frac{\sin (s \pi)}{\pi} \int_{0}^{\infty} \lambda^{-s}\left(1+\lambda+\mathscr{D}^{2}\right)^{-1} \\
& \cdot\left(T+\left[\mathscr{D}^{2}, T\right]\left(1+\mathscr{D}^{2}\right)^{-1}\left(1+\mathscr{D}^{2}\right)\left(1+\lambda+\mathscr{D}^{2}\right)^{-1}\right) d \lambda \\
= & +\left(1+\mathscr{D}^{2}\right)^{s} \frac{\sin (s \pi)}{\pi} \int_{0}^{\infty} \lambda^{-s}\left(1+\lambda+\mathscr{D}^{2}\right)^{-1}\left[\mathscr{D}^{2}, T\right]\left(1+\mathscr{D}^{2}\right)^{-1} \\
& \quad \cdot\left(1+\mathscr{D}^{2}\right)\left(1+\lambda+\mathscr{D}^{2}\right)^{-1} d \lambda .
\end{aligned}
$$

An application of the functional calculus now shows that the integral is norm convergent, but in order to show that $\left(1+\mathscr{D}^{2}\right)^{s}$ times the integral is bounded, we must work a little harder. We write

$$
\left(1+\mathscr{D}^{2}\right)\left(1+\lambda+\mathscr{D}^{2}\right)^{-1}=1-\lambda\left(1+\lambda+\mathscr{D}^{2}\right)^{-1}
$$

so that the integral can be written, with $B=\left[\mathscr{D}^{2}, T\right]\left(1+\mathscr{D}^{2}\right)^{-1}$, as

$$
\begin{aligned}
\int_{0}^{\infty} & \lambda^{-s}\left(1+\lambda+\mathscr{D}^{2}\right)^{-1}\left[\mathscr{D}^{2}, T\right]\left(1+\mathscr{D}^{2}\right)^{-1}\left(1+\mathscr{D}^{2}\right)\left(1+\lambda+\mathscr{D}^{2}\right)^{-1} d \lambda \\
= & \int_{0}^{\infty} \lambda^{-s}\left(1+\lambda+\mathscr{D}^{2}\right)^{-1} B d \lambda \\
& -\int_{0}^{\infty} \lambda^{-s} \lambda\left(1+\lambda+\mathscr{D}^{2}\right)^{-1} B\left(1+\lambda+\mathscr{D}^{2}\right)^{-1} d \lambda .
\end{aligned}
$$

The first integral on the right hand side converges in norm to $\frac{\pi}{\sin (s \pi)}\left(1+\mathscr{D}^{2}\right)^{-s} B$. For the second integral on the right hand side, we suppose first that $B$ is self-adjoint. Then

$\lambda\left(1+\lambda+\mathscr{D}^{2}\right)^{-1} B\left(1+\lambda+\mathscr{D}^{2}\right)^{-1} \leq\|B\| \lambda\left(1+\lambda+\mathscr{D}^{2}\right)^{-2} \leq\|B\|\left(1+\lambda+\mathscr{D}^{2}\right)^{-1}$.

Thus for $B$ self-adjoint, the second integral converges in norm to an operator which is bounded above by $\frac{\pi}{\sin (s \pi)}\left(1+D^{2}\right)^{-s}\|B\|$. By decomposing $B$ into its real and imaginary parts, this is true for any bounded $B$. Thus $\left(1+\mathscr{D}^{2}\right)^{s} T\left(1+\mathscr{D}^{2}\right)^{-s}$ is bounded for $0<s<1$, and a similar argument shows that $\left(1+\mathscr{D}^{2}\right)^{-s} T\left(1+\mathscr{D}^{2}\right)^{s}$ is bounded. 
In all the following, we define $R_{S}(\lambda):=\left(\lambda-\left(1+s^{2}+\mathscr{D}^{2}\right)\right)^{-1}$ for $s \geq 0$ and $\lambda$ in the vertical line

$$
l:=\{a+i v \mid-\infty<v<\infty\}
$$

for some fixed $0<a<1 / 2$.

Lemma 3.3. Let $(\mathcal{A}, \mathscr{H}, \mathcal{D})$ be a weakly $\mathrm{Q} C^{\infty}$ modular spectral triple relative to $(\mathcal{N}, \phi)$. Then $R_{S}(\lambda)\left[\mathscr{D}^{2}, T\right]$ is uniformly bounded on the line l independent of $s, \lambda$ for all $T \in \mathcal{A} \cup[\mathcal{D}, \mathcal{A}]$. For all $T \in \mathcal{A} \cup[\mathcal{D}, \mathcal{A}]$, the function $\lambda \mapsto R_{S}(\lambda) T R_{S}(\lambda)^{-1}$ is uniformly bounded and differentiable on the line $l$ with derivative $-R_{S}(\lambda)^{2}\left[D^{2}, T\right]$, which vanishes as $\lambda \rightarrow a \pm i \infty$.

Proof. First $R_{S}(\lambda)\left[\mathscr{D}^{2}, T\right]=R_{S}(\lambda)\left(1+\mathscr{D}^{2}\right)\left(1+\mathscr{D}^{2}\right)^{-1}\left[\mathscr{D}^{2}, T\right]$ and $R_{s}(\lambda)\left(1+\mathscr{D}^{2}\right)$ is uniformly bounded. Then $R_{S}(\lambda) T R_{S}(\lambda)^{-1}=R_{S}(\lambda)\left[\mathscr{D}^{2}, T\right]+T$ is uniformly bounded on $l$. For the differentiability, we form the difference quotients

$$
\begin{aligned}
& R_{s}(\lambda+\varepsilon) T R_{s}(\lambda+\varepsilon)^{-1}-R_{S}(\lambda) T R_{s}(\lambda)^{-1} \\
& \quad=\left(R_{s}(\lambda+\varepsilon)-R_{s}(\lambda)\right) T R_{S}(\lambda+\varepsilon)^{-1}+R_{s}(\lambda) T\left(R_{s}(\lambda+\varepsilon)-R_{s}(\lambda)^{-1}\right) \\
& \quad=-\varepsilon R_{s}(\lambda+\varepsilon) R_{S}(\lambda) T R_{S}(\lambda+\varepsilon)^{-1}+\varepsilon R_{s}(\lambda) T,
\end{aligned}
$$

where $\varepsilon$ is chosen so that $\lambda+\varepsilon$ lies in a small ball centred on $\lambda=a+i v$. Now the uniform boundedness of $R_{S}(\lambda) T R_{S}(\lambda)^{-1}$ and the boundedness of $R_{S}(\lambda) T$ show that after dividing by $\varepsilon$, the norm limit as $\varepsilon \rightarrow 0$ exists and is given by

$$
R_{S}(\lambda) T-R_{S}(\lambda)^{2} T R_{S}(\lambda)^{-1}=-R_{S}(\lambda)^{2}\left[D^{2}, T\right] .
$$

This is not only bounded but goes to zero as $|\lambda| \rightarrow \infty$ along the line $l=a+i v$.

We recall the notation $T^{(n)}=\left[\mathscr{D}^{2}, T^{(n-1)}\right]$ for $n \geq 1$ and $T^{(0)}:=T$ for $T$ mapping $\mathscr{H}_{\infty}$ to itself.

Lemma 3.4. With $(\mathcal{A}, \mathcal{H}, \mathcal{D})$ as above and $T \in \mathcal{A} \cup[\mathcal{D}, \mathcal{A}]$, we have the formula

$$
R_{S}(\lambda)^{n} T R_{s}(\lambda)^{-n}=T+\sum_{j=1}^{n}(n-j+1) R_{s}(\lambda)^{j} T^{(j)} .
$$

Proof. Induction and the formula $R_{S}(\lambda) T R_{S}(\lambda)^{-1}=T+R_{S}(\lambda)\left[\mathcal{D}^{2}, T\right]$.

Corollary 3.5. The function $\lambda \mapsto R_{S}(\lambda)^{n} T R_{S}(\lambda)^{-n}$ is norm differentiable for all $T \in \mathcal{A} \cup[\mathcal{D}, \mathcal{A}]$. The derivative goes to zero in norm as $\lambda \rightarrow a \pm i \infty$ and is given by

$$
\frac{d}{d \lambda} R_{S}(\lambda)^{n} T R_{S}(\lambda)^{-n}=-R_{S}(\lambda) \sum_{j=1}^{n} j(n-j+1) R_{S}(\lambda)^{j} T^{(j)} .
$$


We now prove the main technical result we require, which weakens the smoothness hypotheses of [CPRS2], Lemma 7.2.

Lemma 3.6. Let $(\mathcal{A}, \mathcal{H}, \mathcal{D}, \mathcal{N}, \phi)$ be a weakly $\mathrm{Q} C^{\infty}$ modular spectral triple of dimension $p \geq 1$. Let $m$ be a non-negative integer and $j=0, \ldots, m$.

(1) Let $A_{j} \in \mathrm{w}-\mathrm{OP}^{k_{j}}, k_{j} \geq 0$, with the product $A_{0} A_{1} \ldots A_{m}$ being $\sigma_{t}^{\phi}$-invariant and affiliated to $\mathcal{M}=\mathcal{N}^{\sigma^{\phi}}$. Then the map

$$
r \mapsto B^{r}(s)=\frac{1}{2 \pi i} \int_{l} \lambda^{-\frac{p}{2}-r} A_{0} R_{S}(\lambda) A_{1} R_{S}(\lambda) A_{2} \ldots R_{s}(\lambda) A_{m} R_{s}(\lambda) d \lambda,
$$

is an analytic function with values in $\operatorname{dom}(\phi)$ for $r \in\left\{z \in \mathbb{C} \mid \operatorname{Re}(z)>\frac{|k|}{2}-m, z \notin\right.$ $\left.\mathbb{N}-\frac{p}{2}\right\}$, where $|k|=k_{0}+k_{1}+\cdots+k_{m}$. For $\alpha>0$, the function $s \mapsto s^{\alpha} \times \phi\left(\left|B^{r}(s)\right|\right)$ is integrable on $[0, \infty)$ when in addition we have $1+\alpha+|k|-2 m<2 \operatorname{Re}(r)$.

(2) Define $\hat{R}_{S}(\lambda)=\left(\lambda-\left(1+s^{2}+D^{2}+s K\right)\right)^{-1}$ for an operator $K=K^{*}$ with $\|K\|_{\infty} \leq \sqrt{2}$. For $a_{j} \in \mathcal{A}$ with $a_{0} a_{1} \ldots a_{m} \in \mathcal{M}$ and $r \in \mathbb{C}$ with $\operatorname{Re}(r)>0$ the operator

$$
\widetilde{B}^{r}(s)=\frac{1}{2 \pi i} \int_{l} \lambda^{-\frac{p}{2}-r} a_{0} R_{s}(\lambda)\left[\mathcal{D}, a_{1}\right] R_{s}(\lambda)\left[\mathcal{D}, a_{2}\right] \ldots R_{s}(\lambda)\left[\mathscr{D}, a_{m}\right] \widehat{R}_{s}(\lambda) d \lambda
$$

is in $\operatorname{dom}(\phi)$, and the function $s \mapsto s^{m} \times \phi\left(\left|\widetilde{B}^{r}(s)\right|\right)$ is integrable on $[0, \infty)$ when $p<1+m$ and $1<m+2 \operatorname{Re}(r)$.

Proof. The restriction of $\phi$ to the fixed point algebra $\mathcal{M}:=\mathcal{N}^{\sigma^{\phi}}$ is a semi-finite trace. By assumption, we have $\left(1+\mathscr{D}^{2}\right)^{-\frac{1}{2}} \in \mathcal{M}$, so $R_{S}(\lambda) \in \mathcal{M}$, and $A_{0} A_{1} \ldots A_{m}$ is affiliated to $\mathcal{M}$. Hence, the estimates in this proof will be done in the von Neumann algebra $\mathcal{M}$, and we denote the trace norm, with respect to $\phi$ on $\mathcal{M}$, by $\|\cdot\|_{1}$.

To prove statement (1), the strategy is to use the fundamental theorem of calculus, at first just doing norm convergence of integrals and norm differentiability. We abbreviate $R:=R_{S}(\lambda)$, fix $k_{0}, \ldots, k_{m}$ as in the statement, and with $\operatorname{Re}(r)>0$ sufficiently large, we have, for any integer $M>m$,

$$
\begin{aligned}
& \frac{1}{2 \pi i} \int_{l} \lambda^{-\frac{p}{2}-r} A_{0} R A_{1} R \ldots R A_{m} R d \lambda \\
&= \frac{1}{2 \pi i} \int_{l} \lambda^{-\frac{p}{2}-r} A_{0} R A_{1} R^{-1} R^{2} A_{2} R^{-2} \ldots R^{m} A_{m} R^{-m} R^{m+1} d \lambda \\
&= \frac{(-1)^{M-m}}{2 \pi i\left(\frac{p}{2}+r-1\right)\left(\frac{p}{2}+r-2\right) \ldots\left(\frac{p}{2}+r-(M-m)\right)} \\
& \quad \int_{l} \frac{d^{M-m}}{d \lambda^{M-m}}\left(\lambda^{-\frac{p}{2}-r+(M-m)}\right) A_{0} R A_{1} R^{-1} R^{2} A_{2} R^{-2} \ldots \\
& \quad \ldots R^{m} A_{m} R^{-m} R^{m+1} d \lambda
\end{aligned}
$$


The resolvent cocycle in twisted cyclic cohomology and a local index formula

$$
\begin{aligned}
= & \frac{\Gamma\left(\frac{p}{2}+r-(M-m)\right)}{2 \pi i \Gamma\left(\frac{p}{2}+r\right)} \int_{l} \lambda^{-\frac{p}{2}-r+(M-m)} \sum_{j=0}^{M-m}\left(\begin{array}{c}
M-m \\
j
\end{array}\right) \\
& \cdot \frac{d^{j}}{d \lambda^{j}}\left(A_{0} R A_{1} R^{-1} R^{2} A_{2} R^{-2} \ldots R^{m} A_{m} R^{-m}\right) \frac{d^{M-m-j}}{d \lambda^{M-m-j}}\left(R^{m+1}\right) d \lambda .
\end{aligned}
$$

Iterating the derivative $\frac{d}{d \lambda}\left(R^{m+1}\right)=-(m+1) R^{m+2}$ yields

$$
\frac{d^{M-m-j}}{d \lambda^{M-m-j}}\left(R^{m+1}\right)=(-1)^{M-m-j}\left(\prod_{n=m+1}^{M+1-j} n\right) R^{M+1-j}
$$

Now we consider

$$
\frac{d^{j}}{d \lambda^{j}}\left(A_{0} R A_{1} R^{-1} R^{2} A_{2} R^{-2} \ldots R^{m} A_{m} R^{-m}\right)
$$

We would like to apply Lemma 3.3 to this term, however recall that each $A_{j} \in \mathrm{w}-\mathrm{OP}^{k_{j}}$ and not $\mathrm{w}-\mathrm{OP}^{0}$. So we rewrite

$$
\begin{aligned}
A_{0} R A_{1} R^{-1} R^{2} A_{2} R^{-2} \ldots R^{m} A_{m} R^{-m} & \\
= & A_{0}\left(1+\mathscr{D}^{2}\right)^{-k_{0} / 2}\left(R\left(1+\mathscr{D}^{2}\right)^{k_{0} / 2} A_{1}\left(1+\mathscr{D}^{2}\right)^{-\left(k_{0}+k_{1}\right) / 2} R^{-1}\right) \\
& \cdot\left(R^{2}\left(1+\mathscr{D}^{2}\right)^{\left(k_{0}+k_{1}\right) / 2} A_{2}\left(1+D^{2}\right)^{-\left(k_{0}+k_{1}+k_{2}\right) / 2} R^{-2}\right) \ldots \\
& \ldots\left(R^{m}\left(1+\mathscr{D}^{2}\right)^{\left(|k|-k_{m}\right) / 2} A_{m}\left(1+\mathscr{D}^{2}\right)^{-\frac{|k|}{2}} R^{-m}\right)\left(1+\mathscr{D}^{2}\right)^{\frac{|k|}{2}} .
\end{aligned}
$$

By definition we have $A_{j}\left(1+\mathscr{D}^{2}\right)^{-k_{j} / 2} \in \mathrm{w}-\mathrm{OP}^{0}$, so using Lemma 3.2, we now find that $\left(1+\mathscr{D}^{2}\right)^{s} A_{j}\left(1+\mathscr{D}^{2}\right)^{-s-k_{j} / 2} \in \mathrm{w}-\mathrm{OP}^{0}$ for all $s \in \mathbb{R}$. Hence, we define

$$
A_{j}^{\prime}:=\left(1+\mathscr{D}^{2}\right)^{\frac{1}{2} \sum_{n=0}^{j-1} k_{n}} A_{j}\left(1+\mathscr{D}^{2}\right)^{-\frac{1}{2} \sum_{n=0}^{j} k_{n}} \in \mathrm{w}-\mathrm{OP}^{0},
$$

so that

$$
\begin{aligned}
& A_{0} R A_{1} R^{-1} R^{2} A_{2} R^{-2} \ldots R^{m} A_{m} R^{-m} \\
& \quad=A_{0}^{\prime} R A_{1}^{\prime} R^{-1} R^{2} A_{2}^{\prime} R^{-2} \ldots R^{m} A_{m}^{\prime} R^{-m}\left(1+D^{2}\right)^{\frac{|k|}{2}} .
\end{aligned}
$$

The purpose of introducing $A_{j}^{\prime}$ is to move all the w-OP ${ }^{k_{j}}$ behaviour into the factor $\left(1+\mathscr{D}^{2}\right)^{\frac{|k|}{2}}$ on the right.

We now invoke Corollary 3.5 and find that each factor $R^{j} A_{j}^{\prime} R^{-j}$ is norm differentiable with respect to $\lambda$. Indeed, by eq. (3.1) we have

$$
\frac{d^{n}}{d \lambda^{n}} R^{j} A_{j}^{\prime} R^{-j}=R^{n} B(s, \lambda)
$$


for $n \geq 0$, and some operator $B(s, \lambda)$ uniformly bounded in $s, \lambda$. So we apply the chain rule to

$$
\begin{array}{r}
\frac{d^{j}}{d \lambda^{j}}\left(A_{0}^{\prime} R A_{1}^{\prime} R^{-1} R^{2} A_{2}^{\prime} R^{-2} \ldots R^{m} A_{m}^{\prime} R^{-m}\right)\left(1+\mathcal{D}^{2}\right)^{\frac{|k|}{2}} \\
=\frac{d^{j}}{d \lambda^{j}}\left(A_{0} R A_{1} R^{-1} R^{2} A_{2} R^{-2} \ldots R^{m} A_{m} R^{-m}\right)
\end{array}
$$

and use eq. (3.4) to compute the derivatives. Then Lemma 3.4 allows us to move each resolvent $R^{n}$ arising from eq. (3.4) to the left, which gives

$$
\frac{d^{j}}{d \lambda^{j}}\left(A_{0} R A_{1} R^{-1} R^{2} A_{2} R^{-2} \ldots R^{m} A_{m} R^{-m}\right)=R^{j} B_{j}\left(A_{0}^{\prime}, \ldots, A_{m}^{\prime}\right)\left(1+D^{2}\right)^{\frac{|k|}{2}},
$$

where $B_{j}\left(A_{0}^{\prime}, \ldots, A_{m}^{\prime}\right) \in \mathrm{w}-\mathrm{OP}^{0}$ is uniformly bounded in $s, \lambda$.

Now we absorb the constants $(-1)^{M-m-j}\left(\prod_{n=m+1}^{M+1-j} n\right)$ from eq. (3.3) into $B_{j}\left(A_{0}^{\prime}, \ldots, A_{m}^{\prime}\right)$ and apply the derivative computations to eq. (3.2), which yields

$$
\begin{aligned}
\frac{1}{2 \pi i} \int_{l} \lambda^{-\frac{p}{2}-r} A_{0} R A_{1} R \ldots R A_{m} R d \lambda & \\
= & \frac{\Gamma\left(\frac{p}{2}+r-(M-m)\right)}{2 \pi i \Gamma\left(\frac{p}{2}+r\right)} \int_{l} \lambda^{-\frac{p}{2}-r+(M-m)} \sum_{j=0}^{M-m} \\
& \cdot\left(\begin{array}{c}
M-m \\
j
\end{array}\right) R^{j} B_{j}\left(A_{0}^{\prime}, \ldots, A_{m}^{\prime}\right)\left(1+\mathcal{D}^{2}\right)^{\frac{|k|}{2}} R^{M+1-j} d \lambda \\
= & \frac{\Gamma\left(\frac{p}{2}+r-(M-m)\right)}{2 \pi i \Gamma\left(\frac{p}{2}+r\right)} \int_{l} \lambda^{-\frac{p}{2}-r+(M-m)} \sum_{j=0}^{M-m} \\
& \cdot\left(\begin{array}{c}
M-m \\
j
\end{array}\right) R^{j} B_{j}\left(A_{0}^{\prime}, \ldots, A_{m}^{\prime}\right) R^{-j}\left(1+\mathcal{D}^{2}\right)^{\frac{|k|}{2}} R^{\frac{|k|}{2}} R^{M+1-\frac{|k|}{2}} d \lambda,
\end{aligned}
$$

where the square roots use the principal branch of log.

For each $j$, the operator $R^{j} B_{j}\left(A_{0}^{\prime}, \ldots, A_{m}^{\prime}\right) R^{-j}$ is uniformly bounded in $s, \lambda$ by Lemma 3.4 and the uniform boundedness of $B_{j}\left(A_{0}^{\prime}, \ldots, A_{m}^{\prime}\right)$. Also, the operator $\left(1+\mathscr{D}^{2}\right)^{\frac{|k|}{2}} R^{\frac{|k|}{2}}$ is uniformly bounded in $s, \lambda$, so we are left with estimating $R^{M+1-\frac{|k|}{2}}$. The trace estimate for the resolvent in [CPRS2], Lemma 5.3, states that for $M$ large enough and all $\varepsilon>0$, there is a constant $C_{\varepsilon}>0$ such that

$$
\left\|R^{M+1-\frac{|k|}{2}}\right\|_{1} \leq C_{\varepsilon}\left(\left(1 / 2+s^{2}-a\right)^{2}+v^{2}\right)^{-\left(M+1-\frac{|k|}{2}\right) / 2+(p / 4+\varepsilon)} .
$$

This estimate and the uniform boundedness of each $R^{j} B_{j}\left(A_{0}^{\prime}, \ldots, A_{m}^{\prime}\right) R^{-j}$, implies that

$$
\frac{1}{2 \pi i} \int_{l} \lambda^{-\frac{p}{2}-r} A_{0} R A_{1} R \ldots R A_{m} R d \lambda \in \operatorname{dom}(\phi)
$$


for $|k|-2 m+\varepsilon<2 \operatorname{Re}(r)$, for all $\varepsilon>0$. We may apply this estimate only when $r \neq(M-j)-\frac{p}{2}$ as the prefactor

$$
\frac{\Gamma\left(\frac{p}{2}+r-(M-m)\right)}{2 \pi i \Gamma\left(\frac{p}{2}+r\right)}=\frac{1}{2 \pi i\left(\frac{p}{2}+r-1\right)\left(\frac{p}{2}+r-2\right) \ldots\left(\frac{p}{2}+r-(M-m)\right)}
$$

has a pole at these points. So now we estimate

$$
\int_{0}^{\infty} s^{\alpha} \phi\left(\frac{1}{2 \pi i} \int_{l} \lambda^{-\frac{p}{2}-r} A_{0} R A_{1} R \ldots R A_{m} R d \lambda\right) d s
$$

in trace norm (recall that we regard $\phi$ as a trace on the fixed point algebra $\mathcal{M}$ ). The calculations above show that the trace norm is bounded by

$$
\begin{aligned}
& \frac{\left|\Gamma\left(\frac{p}{2}+r-(M-m)\right)\right|}{2 \pi\left|\Gamma\left(\frac{p}{2}+r\right)\right|} \int_{0}^{\infty} s^{\alpha} \int_{-\infty}^{\infty}{\sqrt{a^{2}+v^{2}}}^{\frac{p}{2}-\operatorname{Re}(r)+(M-m)} \\
& \cdot \sum_{j=0}^{M-m}\left(\begin{array}{c}
M-m \\
j
\end{array}\right)\left\|R^{j} B_{j}\left(A_{0}^{\prime}, A_{1}^{\prime}, \ldots, A_{m}^{\prime}\right) R^{-j}\right\|_{\infty}\left\|R^{M+1-\frac{|k|}{2}}\right\|_{1} d v d s \\
& \leq \sum_{j=0}^{M-m}\left(\begin{array}{c}
M-m \\
j
\end{array}\right) \frac{\left|\Gamma\left(\frac{p}{2}+r-(M-m)\right)\right|}{2 \pi\left|\Gamma\left(\frac{p}{2}+r\right)\right|} C_{\varepsilon}^{\prime} \int_{0}^{\infty} s^{\alpha} \\
& \cdot \int_{-\infty}^{\infty}{\sqrt{a^{2}+v^{2}}}^{(M-m)-\frac{p}{2}-\operatorname{Re}(r)}{\sqrt{\left(1 / 2+s^{2}-a\right)^{2}+v^{2}}}^{\frac{|k|}{2}-M-1+(p+\varepsilon) / 2} d v d s,
\end{aligned}
$$

where the constant $C_{\varepsilon}^{\prime}$ incorporates the constant from the estimate in eq. (3.5) and the constant coming from $\left\|R^{j} B_{j}\left(A_{0}^{\prime}, A_{1}^{\prime}, \ldots, A_{m}^{\prime}\right) R^{-j}\right\|_{\infty} \leq C$. Now by [CPRS2], Lemma 5.4, the double integral converges for

$$
(\alpha+|k|-M)+(p+\varepsilon-M)<1 \quad \text { and } \quad(\alpha+|k|)-2 m+\varepsilon-2 \operatorname{Re}(r)<-1 .
$$

The first constraint can always be satisfied by taking $M$ sufficiently large. The second holds precisely when $\alpha+|k|+1-2 m<2 \operatorname{Re}(r)$, by choosing $\varepsilon$ small enough.

Statement (2) of the lemma is proved just as above, with the extra $\widehat{R}_{S}(\lambda)$ just estimated in operator norm, using [CPRS2], Lemma 5.1,

$$
\left\|\hat{R}_{s}(\lambda)\right\|_{\infty} \leq\left(v^{2}+\left(1+s^{2}-a-s\|K\|_{\infty}\right)^{2}\right)^{-\frac{1}{2}},
$$

and the general integral estimate [CPRS2], Lemma 5.4.

\subsection{Existence of the resolvent cocycle for weakly $Q C^{\infty}$ modular spectral triples.}

First we explicitly define the resolvent cocycle associated to a modular spectral triple, again just working in the odd case. The definitions in the even case can be deduced from [CPRS3]. 
Definition 3.7. Let $(\mathcal{A}, \mathscr{H}, \mathscr{D}, \mathcal{N}, \phi)$ be a weakly $\mathrm{Q} C^{\infty}$ odd modular spectral triple of dimension $p \geq 1$. Let $N=\left[\frac{p}{2}\right]+1$ be the least integer strictly greater than $\frac{p}{2}$. Let $m$ be an odd integer, $1 \leq m \leq 2 N-1$, and let $A_{j} \in \mathrm{w}_{-}-\mathrm{OP}^{k_{j}}, j=0, \ldots, m$, be operators whose product $A_{0} A_{1} \ldots A_{m}$ is $\sigma_{t}^{\phi}$-invariant and affiliated to $\mathcal{M}$. For $2 \operatorname{Re}(r)>\left(k_{0}+\cdots+k_{m}\right)+1-m, r \notin \mathbb{N}-\frac{p}{2}$, define

$$
\left\langle A_{0}, \ldots, A_{m}\right\rangle_{m, s, r}:=\frac{1}{2 \pi i} \phi\left(\int_{l} \lambda^{-\frac{p}{2}-r} A_{0} R_{s}(\lambda) A_{1} \ldots A_{m} R_{s}(\lambda) d \lambda\right) .
$$

The resolvent cocycle $\left(\Phi_{m}^{r}\right)_{m=1,3, \ldots, 2 N-1}$ is defined to be

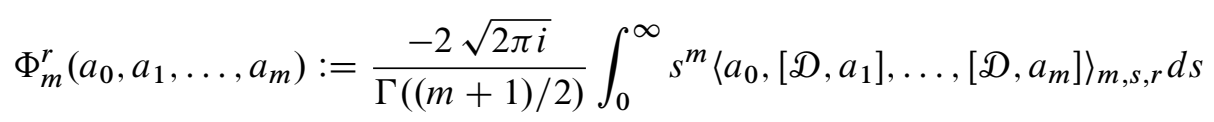

for $a_{i} \in \mathcal{A}$ satisfying $a_{0} a_{1} \ldots a_{m} \in \mathcal{M}$. For brevity we introduce the notation $d a:=[\mathscr{D}, a]$ for $a \in \mathcal{A}$.

We observe that $\Phi_{m}^{r}$ is finite for $\operatorname{Re}(r)>(1-m) / 2$, by Lemma 3.6. In this subsection we show that for weakly smooth modular spectral triples, $\left(\Phi_{m}^{r}\right)_{m=1, \ldots, 2 N-1}$ defines a twisted $b, B$ cocycle modulo functions holomorphic in the half-plane $r>(1-p) / 2$.

We start by presenting the $s$ - and $\lambda$-tricks, which are the main tools needed to prove continuity of the resolvent cocycle. These tricks appeared in [CPRS2], [CPRS3], [CPRS4] without appropriate justification for the convergence of the derivatives in trace norm. In [CGRS2] the justification was given with the aid of the pseudodifferential calculus. Here we present a different proof using only the weak $\mathrm{Q} C^{\infty}$ hypothesis.

Lemma 3.8 (s-trick). Let $(\mathcal{A}, \mathcal{H}, \mathscr{D})$ be a weakly $\mathrm{Q} C^{\infty}$ odd modular spectral triple relative to $(\mathcal{N}, \phi)$ of dimension $p \geq 1$. For any integers $m \geq 0, k \geq 1$ and operators $A_{0}, \ldots, A_{m}$ with $A_{j} \in \mathrm{w}-\mathrm{OP}^{k_{j}}$, and $2 \operatorname{Re}(r)>k+2 \sum k_{j}-2 m, r \notin \mathbb{N}-\frac{p}{2}$, we may choose $r$ with $\operatorname{Re}(r)$ sufficiently large such that

$$
\begin{aligned}
k \int_{0}^{\infty} & s^{k-1}\left\langle A_{0}, \ldots, A_{m}\right\rangle_{m, s, r} d s \\
& =-2 \sum_{j=0}^{m} \int_{0}^{\infty} s^{k+1}\left\langle A_{0}, \ldots, A_{j}, 1, A_{j+1}, \ldots, A_{m}\right\rangle_{m+1, s, r} d s .
\end{aligned}
$$

Proof. The only thing that needs justification is the trace norm derivative formula

$$
\frac{d}{d s}\left\langle A_{0}, \ldots, A_{m}\right\rangle_{m, s, r}=2 s \sum_{k=0}^{m}\left\langle A_{0}, \ldots, A_{k}, 1, A_{k+1}, \ldots, A_{m}\right\rangle_{m+1, s, r}
$$


for suitable $m, s, r$ and weak pseudodifferential operators $A_{j}$. So start with the difference quotient leading to one of the terms on the right hand side.

$$
\begin{aligned}
& \frac{1}{2 \pi i} \int_{l} \lambda^{-\frac{p}{2}-r} A_{0} R \ldots R A_{k}\left(\frac{R_{s+\varepsilon}-R_{s}}{\varepsilon}\right) A_{k+1} R \ldots R A_{m} R d \lambda \\
& \quad=(2 s+\varepsilon) \frac{1}{2 \pi i} \int_{l} \lambda^{-\frac{p}{2}-r} A_{0} R \ldots R A_{k} R_{s+\varepsilon} R_{s} A_{k+1} R \ldots R A_{m} R d \lambda .
\end{aligned}
$$

Now repeat the trick of Lemma 3.6, giving

$$
\begin{aligned}
= & (2 s+\varepsilon) \frac{1}{2 \pi i\left(\frac{p}{2}+r-1\right)\left(\frac{p}{2}+r-2\right) \ldots\left(\frac{p}{2}+r-(2 M-1-m)\right)} \\
& \cdot \int_{l} \lambda^{-\frac{p}{2}-r+(2 M-1-m)} \sum_{j=0}^{2 M-1-m}\left(\begin{array}{c}
2 M-1-m \\
j
\end{array}\right) \frac{d^{j}}{d \lambda^{j}}\left(A_{0} R A_{1} R^{-1} R^{2} A_{2} R^{-2} \ldots\right. \\
& \left.\ldots R^{k} A_{k} R^{-k} R_{s+\varepsilon} R^{k+1} A_{k+1} R^{-k-1} \ldots R^{m} A_{m} R^{-m}\right) \frac{d^{2 M-1-m-j}}{d \lambda^{2 M-1-m-j}}\left(R^{m+1}\right) d \lambda .
\end{aligned}
$$

Performing the derivatives yields a formula similar to that in the proof of Lemma 3.6, but in place of the uniformly bounded $B_{j}$ 's, we have uniformly bounded operators and one extra resolvent. Thus the same trace norm estimates apply and we see that the difference quotients converge in trace norm. Thus $\left\langle A_{0}, \ldots, A_{m}\right\rangle_{m, s, r}$ is trace norm differentiable in $s$, and the derivative goes to zero as $\lambda \rightarrow a \pm i \infty$. The proof is completed by applying the fundamental theorem of calculus to

$$
\frac{d}{d s}\left(s^{k}\left\langle A_{0}, \ldots, A_{m}\right\rangle_{m, s, r}\right) .
$$

A completely analogous argument using the fundamental theorem of calculus for the parameter $\lambda$ proves the following ' $\lambda$-trick' with our weak smoothness hypotheses.

Lemma 3.9 ( $\lambda$-trick). Let ( $\mathcal{A}, \mathcal{H}, \mathcal{D})$ be a weakly $\mathrm{Q} C^{\infty}$ odd modular spectral triple relative to $(\mathcal{N}, \phi)$ of dimension $p \geq 1$. For any integer $m \geq 0$, operators $A_{j} \in$ $\mathrm{w}-\mathrm{OP}^{k_{j}}, j=0, \ldots, m$, and $r$ such that $2 \operatorname{Re}(r)>2 \sum k_{j}-2 m, r \notin \mathbb{N}-\frac{p}{2}$, we have

$$
-\left(\frac{p}{2}+r\right)\left\langle A_{0}, \ldots, A_{m}\right\rangle_{m, s, r+1}=\sum_{k=0}^{m}\left\langle A_{0}, \ldots, A_{k}, 1, A_{k+1}, \ldots, A_{m}\right\rangle_{m+1, s, r} .
$$

Proposition 3.10. Let $(\mathcal{A}, \mathscr{H}, \mathcal{D})$ be a weakly $\mathrm{Q} C^{\infty}$ odd modular spectral triple relative to $(\mathcal{N}, \phi)$ of dimension $p \geq 1$. Let $m=1,3, \ldots, 2 N-1$. Let $\mathcal{A} \otimes$ $\mathcal{A}^{\otimes m}$ have the projective tensor product topology coming from the seminorms $a \mapsto$ $\left\|W R^{k} \circ W L^{l}(a)\right\|_{\infty}+\left\|W R^{k} \circ W L^{l}([\mathcal{D}, a])\right\|_{\infty}$ on $\mathcal{A}$, and restrict this topology to the subspace $\left(\mathcal{A} \otimes \mathcal{A}^{\otimes m}\right)^{\sigma^{\phi}}$ of $\sigma^{\phi}$ invariant tensors. (This can be called the weak $\mathrm{Q} C^{\infty}$-topology). Then the maps

$$
\left(\mathscr{A} \otimes \mathcal{A}^{\otimes m}\right)^{\sigma^{\phi}} \ni a_{0} \otimes \cdots \otimes a_{m} \mapsto\left[r \mapsto \Phi_{m}^{r}\left(a_{0}, \ldots, a_{m}\right)\right]
$$


are continuous multilinear maps from $\left(\mathcal{A} \otimes \mathcal{A}^{\otimes m}\right)^{\sigma^{\phi}}$ to the functions holomorphic in $\left\{z \in \mathbb{C} \mid \operatorname{Re}(z)>(1-m) / 2, z \notin \mathbb{N}-\frac{p}{2}\right\}$, with the topology of uniform convergence on compacta.

Proof. Let us first fix $r \in \mathbb{C}$ with $\operatorname{Re}(r)>(1-m) / 2$, and set $M=2 N-1$. Lemma 3.6 ensures that our functionals are finite for these values of $r$, and it is an exercise (see [CPRS2], Lemma 7.4) to show that these functionals are holomorphic there. Thus all that we need to do is to improve the estimates to prove continuity. We do this, following [CPRS4], Proposition 5.18, using the $s$ - and $\lambda$-tricks. We recall that we have defined $M=2 N-1$. By applying successively the $s$ - and $\lambda$-tricks (which commute) $(M-m) / 2$ times each, we obtain

$$
\begin{aligned}
\Phi_{m}^{r}\left(a_{0}, \ldots, a_{m}\right) & \\
= & 2^{(M-m) / 2}(M-m) ! \prod_{l_{1}=1}^{(M-m) / 2} \frac{1}{\frac{p}{2}+r-l_{1}} \prod_{l_{2}=1}^{(M-m) / 2} \frac{1}{m+l_{2}} \\
& \cdot \sum_{|k|=M-m} \int_{0}^{\infty} s^{M}\left\langle a_{0}, 1^{k_{0}}, d a_{1}, 1^{k_{1}}, \ldots, d a_{m}, 1^{k_{m}}\right\rangle_{M, s, r-(M-m) / 2} d s,
\end{aligned}
$$

where $1^{k_{i}}=1,1, \ldots, 1$ with $k_{i}$ entries. Since $M \leq p+1$, the poles associated to the prefactors are outside the region $\{z \in \mathbb{C} \mid \operatorname{Re}(z)>(1-m) / 2\}$. Ignoring the prefactors, setting $n_{i}=k_{i}+1$ and $R:=R_{s, t}(\lambda)$, we need to deal with the integrals

$$
\int_{0}^{\infty} s^{M} \phi\left(\int_{l} \lambda^{-\frac{p}{2}-r-(M-m) / 2} a_{0} R^{n_{0}} d a_{1} R^{n_{1}} \ldots d a_{m} R^{n_{m}} d \lambda\right) d s, \quad|n|=M+1,
$$

where $l$ is the vertical line $l=\{a+i v \mid v \in \mathbb{R}\}$ with $a=1 / 2$.

To estimate the trace norm (using the trace given by restricting $\phi$ to the invariant subalgebra $\mathcal{N}^{\sigma^{\phi}}$ ) we first write

$$
\begin{aligned}
a_{0} R^{n_{0}} d a_{1} R^{n_{1}} \ldots d a_{m} R^{n_{m}}= & a_{0}\left(R^{n_{0}} d a_{1} R^{-n_{0}}\right)\left(R^{n_{0}+n_{1}} d a_{2} R^{-\left(n_{0}+n_{1}\right)}\right) \ldots \\
& \ldots\left(R^{n_{0}+\cdots+n_{m-1}} d a_{m} R^{-\left(n_{0}+\cdots+n_{m-1}\right)}\right) R^{n_{0}+\cdots+n_{m}} .
\end{aligned}
$$

Then, using [CPRS2], Lemma 5.2, and the fact that $|n|=M+1$, for each $\varepsilon>0$ we obtain $C_{\varepsilon}>0$ such that

$$
\begin{array}{rl}
\| a_{0} R^{n_{0}} & d a_{1} R^{n_{1}} \ldots d a_{m} R^{n_{m}} \|_{1} \\
\leq & \| a_{0}\left(R^{n_{0}} d a_{1} R^{-n_{0}}\right)\left(R^{n_{0}+n_{1}} d a_{2} R^{-\left(n_{0}+n_{1}\right)}\right) \ldots \\
& \ldots\left(R^{n_{0}+\cdots+n_{m-1}} d a_{m} R^{-\left(n_{0}+\cdots+n_{m-1}\right)}\right)\left\|_{\infty}\right\| R^{M+1} \|_{1} \\
\leq & \left\|a_{0} R^{n_{0}} d a_{1} R^{n_{1}} \ldots d a_{m} R^{n_{m}} R^{-(M+1)}\right\|_{\infty} \\
& \cdot C_{\varepsilon}\left(\left(s^{2}+a^{2}\right)+v^{2}\right)^{-(M+1) / 2+(p+\varepsilon) / 4} .
\end{array}
$$


The operator norm of the product yields a constant $C\left(a_{0}, a_{1}, \ldots, a_{m}\right)$ depending on $a_{0}, a_{1}, \ldots, a_{m}$, which varies continuously as the $a_{j}$ vary in a weak $\mathrm{Q} C^{\infty}$ continuous way. Integrating now shows that

$$
\left|\Phi_{m}^{r}\left(a_{0}, \ldots, a_{m}\right)\right| \leq|f(r)| C_{\varepsilon, M, m} C\left(a_{0}, a_{1}, \ldots, a_{m}\right)
$$

for a function $f$ continuous for $\operatorname{Re}(r)>(1-m) / 2, r \notin \mathbb{N}-\frac{p}{2}$ (coming from the prefactor and the integral) and some constant $C_{\varepsilon, M, m}$.

Proposition 3.11. Let $(\mathcal{A}, \mathcal{H}, \mathcal{D})$ be a weakly $\mathrm{Q} C^{\infty}$ odd modular spectral triple relative to $(\mathcal{N}, \phi)$ of dimension $p \geq 1$, and let $N=\left[\frac{p}{2}\right]+1$. The collection of functionals $\Phi^{r}=\left\{\Phi_{m}^{r}\right\}_{m=1}^{2 N-1}, m$ odd, is such that

$$
\begin{aligned}
\left(B^{\sigma} \Phi_{m+2}^{r}+b^{\sigma} \Phi_{m}^{r}\right)\left(a_{0}, \ldots, a_{m+1}\right) & =0, \quad m=1,3, \ldots, 2 N-3, \\
\left(B^{\sigma} \Phi_{1}^{r}\right)\left(a_{0}\right) & =0,
\end{aligned}
$$

where the $a_{i} \in \mathcal{A}, \sigma=\sigma_{i}^{\phi}$ and $b^{\sigma}, B^{\sigma}$ are the twisted coboundary operators of cyclic cohomology. Moreover, there is a $\delta^{\prime}, 0<\delta^{\prime}<1$, such that $b^{\sigma} \Phi_{2 N-1}^{r}\left(a_{0}, \ldots, a_{2 N}\right)$ is a holomorphic function of $r$ for $\operatorname{Re}(r)>-\frac{p}{2}+\frac{\delta^{\prime}}{2}$.

Proof. The proof is just as in [CPRS2], Proposition 7.10, using the formulae for the twisted coboundaries $b^{\sigma}, B^{\sigma}$,

$$
\begin{gathered}
\left(b^{\sigma} \Phi_{m}^{r}\right)\left(a_{0}, a_{1}, \ldots, a_{n}, a_{m+1}\right)=\sum_{k=0}^{n}(-1)^{k} \Phi_{m}^{r}\left(a_{0}, \ldots, a_{k} a_{k+1}, \ldots, a_{m+1}\right) \\
+(-1)^{m+1} \Phi_{m}^{r}\left(\sigma\left(a_{m+1}\right) a_{0}, a_{1}, \ldots, a_{n}\right), \\
\left(B^{\sigma} \Phi_{m}^{r}\right)\left(a_{0}, a_{1}, \ldots, a_{m-1}\right)=\sum_{j=0}^{m-1}(-1)^{(m-1) j} \Phi_{m}^{r}\left(1, \sigma\left(a_{j}\right), \sigma\left(a_{j+1}\right), \ldots\right. \\
\left.\ldots, \sigma\left(a_{m-1}\right), a_{0}, \ldots, a_{j-1}\right),
\end{gathered}
$$

and the twisted tracial property of $\phi$. This yields $B^{\sigma} \Phi_{m+2}^{r}+b^{\sigma} \Phi_{m}^{r}=0$ for $m=$ $1,3, \ldots, 2 N-3$.

In particular, computing the Hochschild coboundary uses

$$
\begin{aligned}
& -\left\langle A_{0}, \ldots,\left[\mathcal{D}^{2}, A_{j}\right], \ldots, A_{m}\right\rangle_{m, s, r} \\
& \quad=\left\langle A_{0}, \ldots, A_{j-1} A_{j}, \ldots, A_{m}\right\rangle_{m-1, s, r}-\left\langle A_{0}, \ldots, A_{j} A_{j+1}, \ldots, A_{m}\right\rangle_{m-1, s, r},
\end{aligned}
$$

and, for $k \geq 1$,

$$
\int_{0}^{\infty} s^{k}\left\langle\mathcal{D} A_{0}, A_{1}, \ldots, A_{m}\right\rangle_{m, s, r} d s=\int_{0}^{\infty} s^{k}\left\langle A_{0}, A_{1}, \ldots, A_{m} \mathscr{D}\right\rangle_{m, s, r} d s .
$$

The first identity follows from observing that

$$
-\left[\mathscr{D}^{2}, A_{j}\right]=R_{s}(\lambda)^{-1} A_{j}-A_{j} R_{s}(\lambda)^{-1}
$$


and cancelling neighbouring $R_{S}(\lambda)$ 's. The second follows by applying the twisted cyclicity of the expectation (see [CPRS2], Lemma 7.7, for the tracial version).

Employing these computations for the top degree term $\Phi_{2 N-1}^{r}$ yields

$$
\begin{aligned}
& \left(b^{\sigma} \Phi_{2 N-1}^{r}\right)\left(a_{0}, \ldots, a_{2 N}\right) \\
& =\frac{-2 \sqrt{2 \pi i}}{\Gamma(N)} \sum_{j=1}^{2 N}(-1)^{j} \int_{0}^{\infty} s^{m}\left\langle a_{0},\left[\mathscr{D}, a_{1}\right], \ldots,\left[\mathscr{D}^{2}, a_{j}\right], \ldots,\left[D, a_{2 N}\right]\right\rangle_{2 N, s, r} d s .
\end{aligned}
$$

Since $\mathcal{A} \subset \mathrm{OP}^{0}$ we have $\left[\mathscr{D}^{2}, \mathcal{A}\right] \subset \mathrm{OP}^{1}$, and then the holomorphicity of $b^{\sigma} \Phi_{2 N-1}^{r}$ follows just as in [CPRS2], Proposition 7.10.

We now specialise to the semi-finite case so that we may relate the resolvent cocycle to the index problem (that is, to compute spectral flow). Proposition 3.11 establishes that the resolvent cocycle is almost a cocycle, so we have the following theorem, proven just as in [CPRS2].

Theorem 3.12. Let $(\mathcal{A}, \mathcal{H}, \mathscr{D})$ be a weakly $\mathrm{Q} C^{\infty}$ odd semi-finite spectral triple relative to $(\mathcal{N}, \tau)$ of dimension $p \geq 1$. Let $N=\left[\frac{p}{2}\right]+1$ be the least positive integer strictly greater than $\frac{p}{2}$ and let $u \in \mathcal{A}$ be unitary. Then

$$
s f_{\tau}\left(D, u^{*} D u\right)=\frac{1}{\sqrt{2 \pi i}} \operatorname{Res}_{r=(1-p) / 2}\left(\sum_{m=1, \text { odd }}^{2 N-1} \Phi_{m}^{r}\left(\mathrm{Ch}_{m}(u)\right)\right),
$$

where $\mathrm{Ch}_{m}(u)$ is defined to be

$$
\mathrm{Ch}_{m}(u)=(-1)^{(m-1) / 2}((m-1) / 2) ! u^{*} \otimes u \otimes \cdots \otimes u^{*} \otimes u, \quad(m+1) \text { entries. }
$$

Proof. This 'resolvent index formula' is proved as in [CPRS2], where the differences for the weak $\mathrm{Q} C^{\infty}$ assumption are detailed above.

Remark. In the even case we have a similar statement with $N=[(p+1) / 2]$ and the sum runs over even integers from $m=0$ to $2 N$; see [CPRS3] for the $\mathrm{Q} C^{\infty}$ case and $[\mathrm{S}]$ for the weakly $\mathrm{Q} C^{\infty}$ case. We provide a more precise statement for the general modular case in Theorem 3.14 below.

3.3. The resolvent index formula for modular spectral triples. Let $(\mathcal{A}, \mathscr{H}, \mathscr{D})$ be a modular spectral triple relative to $(\mathcal{N}, \phi)$ with modular group $\sigma^{\phi}$, of spectral dimension $p \geq 1$, and weakly $\mathrm{Q}^{\infty}$ so that

$$
\mathcal{A} \subset \mathrm{OP}^{0}, \quad[\mathscr{D}, \mathcal{A}] \subset \mathrm{w}-\mathrm{OP}^{0} .
$$

Let $u \in M_{n}(\mathcal{A})$ be unitary, $V: \mathbb{T} \rightarrow M_{n}(\mathbb{C})$ a representation and suppose that $u$ is $\sigma^{\phi} \otimes \operatorname{Ad} V$ invariant. 
Lemma 2.7 constructs a semi-finite spectral triple from $(\mathcal{A}, \mathcal{H}, \mathscr{D})$ and $u$. The semi-finite resolvent index formula, Theorem 3.12, then shows that the resolvent cocycle defined using the trace $\phi \otimes G$ is 'almost' a $b, B$ cocycle, and computes the spectral flow from $\mathscr{D}$ to $u \mathscr{D} u^{*}$.

With $N=\left[\frac{p}{2}\right]+1$, we have

$$
s f_{\phi \otimes G}\left(\mathscr{D} \otimes \operatorname{Id}_{n}, u\left(\mathscr{D} \otimes \operatorname{Id}_{n}\right) u^{*}\right)=\frac{1}{\sqrt{2 \pi i}} \operatorname{Res}_{r=\frac{1-p}{2}} \sum_{m=1, \text { odd }}^{2 N-1}\left(\Phi_{G}\right)_{m}^{r}\left(\operatorname{Ch}_{m}(u)\right),
$$

where $\left(\Phi_{G}\right)_{m}^{r}$ is the resolvent cocycle defined using the trace $\phi \otimes G$. In particular the sum on the right hand side of (3.6) analytically continues to a deleted neighbourhood of $r=(1-p) / 2$ with at worst a simple pole at $r=(1-p) / 2$.

We will compute the $G$ part of the trace, leaving us with a functional defined in terms of $\phi$.

The Chern character of $u$ is defined to be the (infinite) sum $\bigoplus_{j} \mathrm{Ch}_{2 j+1}(u) \in$ $\mathrm{HE}_{2 j+1}\left(M_{N}(\mathcal{A})\right)$, the entire cyclic homology, with

$$
\mathrm{Ch}_{2 j+1}(u)=(-1)^{j} j ! u^{*} \otimes u \otimes \cdots \otimes u^{*} \otimes u \quad(2 j+2) \text { entries. }
$$

Now in [W], Lemma 4.1, Wagner has shown, in a slightly different context, that the map

$$
G_{*}: \bigoplus_{j} \mathrm{HE}_{2 j+1}\left(M_{N}(\mathcal{A})^{\sigma \otimes \mathrm{Ad} V}\right) \rightarrow \bigoplus_{j} \mathrm{HE}_{2 j+1}^{\sigma}(\mathcal{A})
$$

to $\sigma$-twisted cyclic homology given on chains by

$$
\begin{aligned}
& G_{*}\left(T_{0} \otimes \cdots \otimes T_{2 j+1}\right) \\
& \quad=\sum_{i_{0}, i_{1}, \ldots, i_{2 j+2}}\left(V_{-i}\right)_{i_{2 j+2}, i_{0}}\left(T_{0}\right)_{i_{0}, i_{1}} \otimes\left(T_{1}\right)_{i_{1}, i_{2}} \otimes \cdots \otimes\left(T_{2 j+1}\right)_{i_{2 j+1}, i_{2 j+2}}
\end{aligned}
$$

is an isomorphism. Here $V_{-i}$ is the extension of the representation of $\mathbb{T}$ to $-i$, as discussed in Section 2.2.

Now each equivariant unitary with class $[u] \in K_{1}^{\mathbb{T}}(A)$ is equivariant for its own representation of the circle. So it makes sense to regard the representation $V$ as part of the data, so $[u]=[u, V]$. We define $\mathrm{Ch}_{2 j+1}([u, V]) \in \mathrm{HE}_{2 j+1}^{\sigma}(\mathcal{A})$ by

$$
\begin{aligned}
& \mathrm{Ch}_{2 j+1}([u, V]) \\
& \quad=(-1)^{j} j ! \sum_{I}\left(V_{-i}\right)_{i_{2 j+2}, i_{0}}\left(u^{*}\right)_{i_{0}, i_{1}} \otimes(u)_{i_{1}, i_{2}} \otimes \cdots \otimes\left(u_{2 j+1}\right)_{i_{2 j+1}, i_{2 j+2}} .
\end{aligned}
$$

Then it is straightforward to check that this does indeed define an entire twisted cyclic 
cycle. Moreover it is immediate from the definitions that

$$
\begin{aligned}
s f_{\phi \otimes G}\left(\mathcal{D} \otimes \operatorname{Id}_{n}, u\left(\mathscr{D} \otimes \operatorname{Id}_{n}\right) u^{*}\right) & =\frac{1}{\sqrt{2 \pi i}} \operatorname{Res}_{r=\frac{1-p}{2}} \sum_{m=1, \text { odd }}^{2 N-1}\left(\Phi_{G}\right)_{m}^{r}\left(\operatorname{Ch}_{m}(u)\right), \\
& =\frac{1}{\sqrt{2 \pi i}} \operatorname{Res}_{r=\frac{1-p}{2}} \sum_{m=1, \text { odd }}^{2 N-1} \Phi_{m}^{r}\left(\operatorname{Ch}_{m}([u, V])\right) .
\end{aligned}
$$

Here $\Phi_{m}^{r}$ is the resolvent cocycle given by the modular spectral triple.

We now collect these observations into a statement describing the resolvent index formula for weakly smooth modular spectral triples.

Theorem 3.13. For a weakly $\mathrm{Q} C^{\infty}$ odd modular spectral triple $(\mathcal{A}, \mathcal{H}, \mathcal{D})$ relative to $(\mathcal{N}, \phi)$ of spectral dimension $p \geq 1$, and with $N=\left[\frac{p}{2}\right]+1$, the function valued cochain $\left(\Phi_{m}^{r}\right)_{m=1, \ldots, 2 N-1}$ is a twisted cyclic cocycle modulo cochains with values in functions holomorphic in a half-plane containing $(1-p) / 2$. Moreover, for $[u, V] \in$ $K_{1}^{\mathrm{T}}(\mathcal{A})$ with representative $u \in M_{n}(\mathcal{A})$ we have

$s f_{\phi \otimes G}\left(\mathscr{D} \otimes \operatorname{Id}_{n}, u^{*}\left(\mathscr{D} \otimes \operatorname{Id}_{n}\right) u\right)=\frac{1}{\sqrt{2 \pi i}} \operatorname{Res}_{r=\frac{1-p}{2}}\left(\sum_{m=1, o d d}^{2 N-1} \Phi_{m}^{r}\left(\operatorname{Ch}_{m}([u, V])\right)\right)$.

In particular, there is a well-defined map

$$
K_{1}^{\mathbb{T}}(\mathcal{A}) \mapsto \mathbb{R}, \quad[u, V] \mapsto s f_{\phi \otimes G}\left(\mathcal{D} \otimes \operatorname{Id}_{n}, u^{*}\left(\mathcal{D} \otimes \operatorname{Id}_{n}\right) u\right) .
$$

Though we have not proved it here, a similar result is true in the even case; see [S].

Theorem 3.14. For a weakly $\mathrm{Q} C^{\infty}$ even modular spectral triple $(\mathcal{A}, \mathscr{H}, \mathcal{D}, \gamma)$ relative to $(\mathcal{N}, \phi)$ of spectral dimension $p \geq 1$, and with $M=[(p+1) / 2]$, the function valued cochain $\left(\Phi_{m}^{r}\right)_{m=0, \ldots, 2 M}$ is a twisted cyclic cocycle modulo cochains with values in functions holomorphic in a half-plane containing $(1-p) / 2$. Moreover, for $[P, V] \in K_{0}^{\mathbb{T}}(\mathcal{A})$ with representative $P \in M_{n}(\mathcal{A})$ and $\mathscr{D}_{+}=\frac{1}{4}(1-\gamma) \mathcal{D}(1+\gamma)$ we have

$\operatorname{Index}_{\phi \otimes G}\left(P\left(D_{+} \otimes \operatorname{Id}_{n}\right) P\right)=\frac{1}{\sqrt{2 \pi i}} \operatorname{Res}_{r=(1-p) / 2}\left(\sum_{m=0, \text { even }}^{2 M} \Phi_{m}^{r}\left(\operatorname{Ch}_{m}([P, V])\right)\right)$.

In particular, there is a well-defined map

$$
K_{0}^{\mathbb{T}}(\mathcal{A}) \mapsto \mathbb{R}, \quad[P, V] \mapsto \operatorname{Index}_{\phi \otimes G}\left(P\left(D_{+} \otimes \operatorname{Id}_{n}\right) P\right) .
$$

Remark. The Chern character of an equivariant projection is

$$
\begin{aligned}
\mathrm{Ch}_{0}([P, V]) & =\operatorname{Tr}\left(V_{-i} P\right), \\
\mathrm{Ch}_{2 k}([P, V]) & =(-1)^{k} \frac{(2 k) !}{k !} \sum\left(V_{-i}\left(P-\frac{1}{2}\right)\right)_{i_{0} i_{1}} \otimes P_{i_{1} i_{2}} \otimes \cdots \otimes P_{i_{2 k} i_{0}} .
\end{aligned}
$$


Finally, the next two results relate the even index given by the resolvent index formula above back to the K-theory valued index pairing between the KK-class defined by the modular spectral triple and equivariant K-theory.

Lemma 3.15. Let $(\mathcal{A}, \mathcal{H}, \mathcal{D})$ be a modular spectral triple relative to $(\mathcal{N}, \phi)$. Let $J_{\phi} \subset \mathcal{N}$ be the ideal from Definition 2.4 and $J_{\phi}^{\sim}$ its unitisation. Let $E \in M_{k}\left(J_{\phi}^{\sim}\right)$ be a $\sigma^{\phi} \otimes \operatorname{Ad} W$-invariant projection for the associated representation $W: \mathbb{T} \rightarrow M_{k}(\mathbb{C})$ so that $[E, W] \in K_{0}^{\mathbb{T}}\left(J_{\phi}^{\sim}\right)$. Define

$$
\phi_{*}([E, W]):=\left(\phi \otimes G_{W}\right)(E) \in[0, \infty],
$$

where $G_{W}(T)=\operatorname{Tr}\left(W_{-i} T\right)$ for $T \in M_{k}(\mathbb{C})$. Then $\phi_{*}$ is a well-defined map on the semigroup of Murray-von Neumann equivalence classes of equivariant projections in $J_{\phi}^{\sim} \otimes \mathcal{K}$, where $\mathcal{K}$ is the compact operators. The Grothendieck group of the subsemigroup for which $\phi_{*}$ takes finite values is (isomorphic to) a subgroup of $K_{0}^{\mathbb{T}}\left(J_{\phi}\right)$, and we call this the domain of $\phi_{*}$.

Proof. Let $W_{1}: \mathbb{T} \rightarrow M_{n}(\mathbb{C})$ and $W_{2}: \mathbb{T} \rightarrow M_{m}(\mathbb{C})$ be representations. Let $E_{1} \in$ $M_{n}\left(J_{\phi}\right)$ denote a $\sigma \otimes$ Ad $W_{1}$ projection, and let $E_{2} \in M_{m}\left(J_{\phi}\right)$ denote a $\sigma^{\phi} \otimes$ Ad $W_{2}$ projection. Suppose that $\left[E_{1}, W_{1}\right]$ and $\left[E_{2}, W_{2}\right]$ are equivariantly Murray-von Neumann equivalent ([W], Definition 3.1), meaning there exists some $S \in M_{m \times n}\left(J_{\phi}\right)$ such that

$$
S^{*} S=E_{1}, \quad S S^{*}=E_{2} \quad \text { and } \quad W_{2, z} S=S W_{1, z} \quad \text { for all } z \in \mathbb{T} .
$$

Then we compute

$$
\begin{aligned}
\phi_{*}\left(\left[E_{1}, W_{1}\right]\right) & =\left(\phi \otimes G_{W_{1}}\right)\left(E_{1}\right) \\
& =\phi\left(\operatorname{Tr}_{n}\left(W_{1,-i} E_{1}\right)\right) \\
& =\phi\left(\operatorname{Tr}_{n}\left(W_{1,-i} S^{*} S\right)\right) \\
& =\phi\left(\operatorname{Tr}_{n}\left(S W_{1,-i} S^{*}\right)\right) .
\end{aligned}
$$

Now, by analytically continuing, $S W_{1,-i}=W_{2,-i} S$, so

$$
\phi_{*}\left(\left[E_{1}, W_{1}\right]\right)=\phi\left(\operatorname{Tr}_{m}\left(W_{2,-i} S S^{*}\right)\right)=\phi\left(\operatorname{Tr}_{m}\left(W_{2,-i} E_{2}\right)\right)=\phi_{*}\left(\left[E_{2}, W_{2}\right]\right) .
$$

Using the universal property of the Grothendieck group, we see that the Grothendieck group of equivalence classes for which $\phi_{*}$ takes finite values may be regarded as a subgroup of $K_{0}^{\mathbb{T}}\left(J_{\phi}\right)$. On this subgroup, $\phi_{*}$ is well defined.

Theorem 3.16. Let $(\mathcal{A}, \mathcal{H}, \mathcal{D}, \gamma)$ be a weakly $\mathrm{Q} C^{\infty}$ even modular spectral triple relative to $(\mathcal{N}, \phi)$ of spectral dimension $p \geq 1$, and $[P, V] \in K_{0}^{\mathbb{T}}(A)$. Let $B_{\phi} \subset J_{\phi}$ be as in Definition 2.5, and let $i: B_{\phi} \rightarrow J_{\phi}$ be the inclusion. Then $i_{*}\left([P, V] \otimes_{A}\right.$ $\left.\left[\left(B_{\phi}, F_{\mathscr{D}}\right)\right]\right) \in K_{0}^{\mathbb{T}}\left(J_{\phi}\right)$ is in the domain of $\phi_{*}$. Furthermore,

$$
\phi_{*}\left(i_{*}\left([P, V] \otimes_{A}\left[\left(B_{\phi}, F_{\mathcal{D}}\right)\right]\right)\right)=\operatorname{Res}_{r=(1-p) / 2}\left(\sum_{m=0, \text { even }}^{2 N} \Phi_{m}^{r}\left(\operatorname{Ch}_{m}([P, V])\right)\right) .
$$


Proof. Given the modular spectral triple $(\mathcal{A}, \mathcal{H}, \mathscr{D}, \gamma, \mathcal{N}, \phi)$, we define $\left[\left(B_{\phi}, F_{\mathscr{D}}\right)\right] \in$ $\mathrm{KK}^{0, \mathbb{T}}\left(A, B_{\phi}\right)$. Also, let $V: \mathbb{T} \rightarrow M_{n}(\mathbb{C})$ be a representation and $P \in M_{n}(\mathcal{A})$ a projection which is $\sigma^{\phi} \otimes \operatorname{Ad} V$ invariant, so that we obtain a class $[P, V] \in K_{0}^{\mathbb{T}}(A)$.

Define the projections

$$
N_{ \pm}:=\operatorname{ker}\left(P\left(\mathscr{D} \otimes \operatorname{Id}_{n}\right)^{ \pm} P\right)
$$

so that

$$
\operatorname{Ind}_{\phi \otimes G}\left(P\left(D \otimes \operatorname{Id}_{n}\right)^{+} P\right)=(\phi \otimes G)\left(N_{+}\right)-(\phi \otimes G)\left(N_{-}\right) .
$$

By the construction of the semi-finite spectral triple $\left(C^{\infty}(P), \mathscr{H} \otimes \mathbb{C}^{n}, \mathscr{D} \otimes \operatorname{Id}_{n}, \mathcal{M}_{n}\right.$, $\phi \otimes G)$, we have $N_{ \pm} \in \mathcal{K}\left(\left(M_{n}(\mathcal{N})\right)^{\sigma^{\phi} \otimes \operatorname{Ad} V}, \phi \otimes G\right)$, since the $N_{ \pm}$are kernel projections and

$$
N_{ \pm} \leq\left(P+\left(P\left(\mathscr{D} \otimes \operatorname{Id}_{n}\right) P\right)^{2}\right)^{-1} .
$$

Also, the $\sigma^{\phi} \otimes$ Ad $V$-invariance of $P$ implies the same invariance for $N_{ \pm}$.

We now want to show that we also have $N_{ \pm} \in M_{n}\left(B_{\phi}\right)$ so that they define classes in $K_{0}^{\mathbb{T}}\left(B_{\phi}\right)$. We do this by proving that the operator $\left(P+\left(P\left(\mathscr{D} \otimes \operatorname{Id}_{n}\right) P\right)^{2}\right)^{-1} \in$ $M_{n}\left(B_{\phi}\right)$, then applying eq. (3.8) again to see that $N_{ \pm} \in M_{n}\left(B_{\phi}\right)$.

Let $\mathscr{D}_{n}:=\operatorname{Id}_{n} \otimes \mathscr{D}$. Consider the operator $\left(P+\left(P \mathscr{D}_{n} P\right)^{2}\right)^{-1}: P\left(\mathbb{C}^{n} \otimes\right.$ $\left.B_{\phi}\right) \rightarrow P\left(\mathbb{C}^{n} \otimes B_{\phi}\right)$. The inverse exists because $P$ acts as the identity on $P\left(\mathbb{C}^{n} \otimes\right.$ $\left.B_{\phi}\right)$ and $\left(P \mathscr{D}_{n} P\right)^{2} \geq 0$. The adjointable endomorphisms on $P\left(\mathbb{C}^{n} \otimes B_{\phi}\right)$ are $P M_{n}\left(M\left(B_{\phi}\right)\right) P$, where $M\left(B_{\phi}\right)$ is the multiplier algebra, while the compact operators are $P M_{n}\left(B_{\phi}\right) P$. A priori, we know only that $\left(P+\left(P D_{n} P\right)^{2}\right)^{-1}$ is bounded on $P\left(\mathbb{C}^{n} \otimes B_{\phi}\right)$.

To show the compactness of $\left(P+\left(P \mathscr{D}_{n} P\right)^{2}\right)^{-1}$, we compute

$$
\begin{aligned}
\left(P+\left(P \mathscr{D}_{n} P\right)^{2}\right)^{-1}= & \left(P+P\left[\mathscr{D}_{n}, P\right] \mathscr{D}_{n} P+P \mathscr{D}_{n}^{2} P\right)^{-1} \\
= & \left(P+P \mathscr{D}_{n}^{2} P\right)^{-1}+\left[\left(P+P\left[\mathscr{D}_{n}, P\right]\left[\mathscr{D}_{n}, P\right] P\right.\right. \\
& \left.\left.+P \mathscr{D}_{n}^{2} P\right)^{-1}-\left(P+P \mathscr{D}_{n}^{2} P\right)^{-1}\right],
\end{aligned}
$$

where the last line follows from the observation

$$
P\left[\mathscr{D}_{n}, P\right] P=P\left[\mathscr{D}_{n}, P^{2}\right] P=P\left(P\left[\mathscr{D}_{n}, P\right]+\left[\mathscr{D}_{n}, P\right] P\right) P=2 P\left[\mathscr{D}_{n}, P\right] P=0,
$$

so that $P\left[\mathscr{D}_{n}, P\right]\left[\mathscr{D}_{n}, P\right] P=P\left[\mathscr{D}_{n}, P\right] \mathscr{D}_{n} P$. The algebraic result $\alpha^{-1}-\beta^{-1}=$ $\beta^{-1}(\beta-\alpha) \alpha^{-1}$ yields

$$
\begin{aligned}
(P+ & \left.P\left[\mathscr{D}_{n}, P\right]\left[\mathscr{D}_{n}, P\right] P+P \mathscr{D}_{n}^{2} P\right)^{-1}-\left(P+P \mathcal{D}_{n}^{2} P\right)^{-1} \\
= & -\left(P+P \mathcal{D}_{n}^{2} P\right)^{-1}\left(P\left[\mathscr{D}_{n}, P\right]\left[\mathscr{D}_{n}, P\right] P\right) \\
& \cdot\left(P+P\left[\mathscr{D}_{n}, P\right]\left[\mathscr{D}_{n}, P\right] P+P \mathscr{D}_{n}^{2} P\right)^{-1} .
\end{aligned}
$$

Hence

$$
\left(P+\left(P \mathscr{D}_{n} P\right)^{2}\right)^{-1}=\left(P+P \mathscr{D}_{n}^{2} P\right)^{-1} B(P),
$$


where $B(P)$ is a bounded operator given by

$$
B(P)=1-\left(P\left[\mathscr{D}_{n}, P\right]\left[\mathscr{D}_{n}, P\right] P\right)\left(P+P\left[\mathscr{D}_{n}, P\right]\left[\mathscr{D}_{n}, P\right] P+P \mathscr{D}_{n}^{2} P\right)^{-1} .
$$

Now consider $\left(1+\mathscr{D}_{n}\right)^{-1}: \mathbb{C}^{n} \otimes B_{\phi} \rightarrow \mathbb{C}^{n} \otimes B_{\phi}$. Then we have

$$
\begin{aligned}
\left(P+P D_{n}^{2} P\right) P\left(1+\mathscr{D}_{n}\right)^{-1} P & =P+P\left[\mathscr{D}_{n}^{2}, P\right]\left(1+\mathscr{D}_{n}^{2}\right)^{-1} P \\
& =P+P C(P)\left(1+\mathscr{D}_{n}^{2}\right)^{-\frac{1}{2}} P .
\end{aligned}
$$

Here $C(P)$ is bounded since $P \in M_{n}(\mathcal{A}) \subset \mathrm{OP}^{0}$ (where $\mathrm{OP}^{0}$ is defined using $\mathscr{D}_{n}$ ).

Now $\left(1+D^{2}\right)^{-\frac{1}{2}} \in B_{\phi}$ by definition, so $\left(1+\mathscr{D}_{n}^{2}\right)^{-\frac{1}{2}} \in M_{n}\left(B_{\phi}\right)$. Hence

$$
P\left[\mathscr{D}_{n}^{2}, P\right]\left(1+\mathscr{D}_{n}^{2}\right)^{-1} P \in P M_{n}\left(B_{\phi}\right) P,
$$

so eq. (3.9) now implies that

$$
\left(P+P D_{n}^{2} P\right)^{-1} \in P M_{n}\left(B_{\phi}\right) P .
$$

We know $B_{\phi}$ is an ideal in the endomorphisms, so eq. (3.8) now implies that $N_{ \pm} \in M_{n}\left(B_{\phi}\right)$. By the $\sigma^{\phi} \otimes \operatorname{Ad} V$-invariance of $N_{ \pm}$, we have $\left[N_{ \pm}, V\right] \in K_{0}^{\mathbb{T}}\left(B_{\phi}\right)$. Then

$$
(\phi \otimes G)\left(N_{+}\right)-(\phi \otimes G)\left(N_{-}\right)=\phi_{*}\left(i_{*}\left(\left[N_{+}, V\right]-\left[N_{-}, V\right]\right)\right) .
$$

In order to compare eq. (3.10) to the Kasparov product $[P, V] \otimes_{A}\left[\left(B_{\phi}, F_{\mathscr{D}}\right)\right]$, we rewrite the classes $\left[N_{ \pm}, V\right]$ as Kasparov modules. We have

$$
\left[N_{+}, V\right]-\left[N_{-}, V\right]=\left[\left(N_{+}\left(\mathbb{C}^{n} \otimes B_{\phi}\right) \oplus N_{-}\left(\mathbb{C}^{n} \otimes B_{\phi}\right), 0,\left(\begin{array}{cc}
N_{+} & 0 \\
0 & -N_{-}
\end{array}\right), V \oplus V\right)\right],
$$

where $N_{+}\left(\mathbb{C}^{n} \otimes B_{\phi}\right) \oplus N_{-}\left(\mathbb{C}^{n} \otimes B_{\phi}\right)$ is the right Hilbert $B_{\phi}$-module, 0 is the operator, $\left(\begin{array}{cc}N_{+} & 0 \\ 0 & -N_{-}\end{array}\right)$is the grading and $V \oplus V$ is the $\mathbb{T}$-action giving the equivariance.

Now the operator $P\left(\operatorname{Id}_{n} \otimes F_{\mathscr{D}}\right)^{+} P$ gives an isomorphism from $\left(1-N_{+}\right)\left(\mathbb{C}^{n} \otimes B_{\phi}\right)$ to $\left(1-N_{-}\right)\left(\mathbb{C}^{n} \otimes B_{\phi}\right)$. Hence, the Kasparov module constructed from $\left(1-N_{ \pm}\right)\left(\mathbb{C}^{n} \otimes\right.$ $\left.B_{\phi}\right)$ and $P\left(\operatorname{Id}_{n} \otimes F_{\mathscr{D}}\right)^{+} P$ has trivial class. Consequently,

$$
\begin{gathered}
{\left[\left(N_{+}\left(\mathbb{C}^{n} \otimes B_{\phi}\right) \oplus N_{-}\left(\mathbb{C}^{n} \otimes B_{\phi}\right), 0,\left(\begin{array}{cc}
N_{+} & 0 \\
0 & -N_{-}
\end{array}\right), V \oplus V\right)\right]} \\
=\left[\left(\mathbb{C}^{n} \otimes B_{\phi}, P\left(\operatorname{Id}_{n} \otimes F_{\mathscr{D}}\right) P, \mathrm{Id}_{n} \otimes \gamma, V\right)\right] .
\end{gathered}
$$

Finally, observe that, see [B] for example, we have an explicit representative of the Kasparov product

$$
[P, V] \otimes_{A}\left[\left(B_{\phi}, F_{\mathscr{D}}\right)\right]=\left[\left(\mathbb{C}^{n} \otimes B_{\phi}, P\left(\operatorname{Id}_{n} \otimes F_{\mathscr{D}}\right) P, \operatorname{Id}_{n} \otimes \gamma, V\right)\right] .
$$

Reiterating the above results, we have proved that

$$
\begin{aligned}
\operatorname{Ind}_{\phi \otimes G}\left(P\left(D \otimes \operatorname{Id}_{n}\right)^{+} P\right) & =(\phi \otimes G)\left(N_{+}\right)-(\phi \otimes G)\left(N_{-}\right) \\
& =\phi_{*}\left(i_{*}\left(\left[N_{+}, V\right]-\left[N_{-}, V\right]\right)\right) \\
& =\phi_{*}\left(i_{*}\left(\left[\left(\mathbb{C}^{n} \otimes B_{\phi}, P\left(\operatorname{Id}_{n} \otimes F_{\mathscr{D}}\right) P, \operatorname{Id}_{n} \otimes \Gamma, V\right)\right]\right)\right) \\
& =\phi_{*}\left(i_{*}\left([P, V] \otimes_{A}\left[\left(B_{\phi}, F_{\mathscr{D}}\right)\right]\right)\right) .
\end{aligned}
$$




\section{The local index formula for the Podleś sphere}

In this section we will explicitly compute a twisted $b, B$ cocycle for the (modular) spectral triple over the Podleś sphere first investigated in [DS]. We do this by applying the modified pseudodifferential calculus of [NT] to the twisted resolvent cocycle of the previous section. Having done this, we construct some equivariant projections for a circle action arising from the Haar state and compute the index pairing via a residue formula, yielding a local index formula.

4.1. The modular spectral triple for the Podleś sphere. We first recall (see [KS]) that the quantum algebra $\mathcal{A}=\mathcal{O}\left(\mathrm{SU}_{q}(2)\right)$ for $q \in[0,1]$ is generated by elements $a$, $b, c, d$ modulo the relations

$$
\begin{gathered}
a b=q b a, \quad a c=q c a, \quad b d=q d b, \quad c d=q d c, \quad b c=c b \\
a d=1+q b c, \quad d a=1+q^{-1} b c \\
a^{*}=d, \quad b^{*}=-q c, \quad c^{*}=-q^{-1} b, \quad d^{*}=a .
\end{gathered}
$$

The Podleś sphere, which we denote by $\mathcal{B}$, is (isomorphic to) the unital $*$ subalgebra of $\mathcal{O}\left(\mathrm{SU}_{q}(2)\right)$ generated by $q^{-1} a b,-c d$ and $-q^{-1} b c$.

Recall that for each $l \in \frac{1}{2} \mathbb{N}_{0}$, there is a unique (up to unitary equivalence) irreducible corepresentation $V_{l}$ of the coalgebra $\mathcal{A}$ of dimension $2 l+1$, and that $\mathcal{A}$ is cosemisimple. That is, if we fix a vector space basis in each of the $V_{l}$ and denote by $t_{r, s}^{l} \in \mathcal{A}$ the corresponding matrix coefficients, then we have the following analogue of the Peter-Weyl theorem.

Theorem 4.1 ([KS], Theorem 4.13). Let $I_{l}:=\{-l,-l+1, \ldots, l-1, l\}$. Then the set $\left\{t_{r, s}^{l} \mid l \in \frac{1}{2} \mathbb{N}_{0}, r, s \in I_{l}\right\}$ is a vector space basis of $\mathcal{A}$.

This will be referred to as the Peter-Weyl basis. With a suitable choice of basis in $V_{\frac{1}{2}}$, one has

$$
a=t_{-\frac{1}{2},-\frac{1}{2}}^{\frac{1}{2}}, \quad b=t_{-\frac{1}{2}, \frac{1}{2}}^{\frac{1}{2}}, \quad c=t_{\frac{1}{2},-\frac{1}{2}}^{\frac{1}{2}}, \quad d=t_{\frac{1}{2}, \frac{1}{2}}^{\frac{1}{2}} .
$$

The expressions for the Peter-Weyl basis elements as linear combinations of the polynomial basis elements can be found in [KS], Section 4.2.4.

The algebra $\mathcal{A}$ has a useful direct sum decomposition. For $m, n \in \mathbb{Z}$ where $m-n$ is even, define

$$
\begin{aligned}
& \mathcal{A}[m, n]:=\operatorname{span}\left\{a^{\frac{1}{2}(m+n)} b^{k+\frac{1}{2}(m-n)} c^{k},\right. \\
& \left.b^{k+\frac{1}{2}(m-n)} c^{k} d^{-\frac{1}{2}(m+n)} \mid k+\min \left\{0, \frac{1}{2}(m-n)\right\} \in \mathbb{N}_{0}\right\},
\end{aligned}
$$

and for $m-n$ odd let $\mathcal{A}[m, n]:=\{0\}$. Then

$$
\mathcal{A}=\bigoplus_{m, n \in \mathbb{Z}} \mathcal{A}[m, n] \quad \text { and } \quad \mathcal{A}\left[m_{1}, n_{1}\right] \cdot \mathcal{A}\left[m_{2}, n_{2}\right] \subseteq \mathcal{A}\left[m_{1}+m_{2}, n_{1}+n_{2}\right] .
$$


With this notation, we have $\mathscr{B}=\bigoplus_{m \in \mathbb{Z}} \mathcal{A}[m, 0]$.

Let $h$ be the Haar state on the universal $C^{*}$-completion of the $*$-algebra $\mathcal{A}$, whose value on the Peter-Weyl basis is $h\left(t_{r, s}^{l}\right)=\delta_{l, 0}$. Define an automorphism $\vartheta$ on $\mathcal{A}$ by

$$
\vartheta(a)=q^{2} a, \quad \vartheta(b)=b, \quad \vartheta(c)=c, \quad \vartheta(d)=q^{-2} d .
$$

Then $\vartheta$ is the modular automorphism for the Haar state, in the sense that $h(\alpha \beta)=$ $h(\vartheta(\beta) \alpha)$ for all $\alpha, \beta \in \mathcal{A}$. For all $n \in \mathbb{Z}$ define

$$
\mathscr{H}_{n}:=L^{2}\left(\operatorname{span}\left\{t_{r, \frac{n}{2}}^{l} \mid l \in \frac{n}{2}+\mathbb{N}_{0}, r \in I_{l}\right\}, h\right) .
$$

The left action of the dual Hopf algebra to $\mathcal{A}$ provides the unbounded operators $\partial_{e}: \mathscr{H}_{n} \rightarrow \mathscr{H}_{n+2}$ and $\partial_{f}: \mathscr{H}_{n} \rightarrow \mathscr{H}_{n-2}$ given by

$$
\partial_{e}\left(t_{r, s}^{l}\right)=\sqrt{\left[l+\frac{1}{2}\right]_{q}^{2}-\left[s+\frac{1}{2}\right]_{q}^{2}} t_{r, s+1}^{l}, \quad \partial_{f}\left(t_{r, s}^{l}\right)=\sqrt{\left[l+\frac{1}{2}\right]_{q}^{2}-\left[s-\frac{1}{2}\right]_{q}^{2}} t_{r, s-1}^{l},
$$

where our definition of the $q$-number $[a]_{q}$ is

$$
[a]_{q}:=\frac{q^{-a}-q^{a}}{q^{-1}-q}=Q\left(q^{-a}-q^{a}\right) \text { for any } a \in \mathbb{C},
$$

and we abbreviated $Q:=\left(q^{-1}-q\right)^{-1} \in(0, \infty)$. Finally, we define unbounded linear operators $\Delta_{R}, \partial_{k}$ on $\mathscr{A}_{\mathcal{A}} \subset \bigoplus \mathscr{H}_{n}$ by

$$
\Delta_{R}\left(t_{r, s}^{l}\right):=q^{2 r} t_{r, s}^{l}, \quad \partial_{k} \triangleright t_{i, j}^{l}:=q^{j} t_{i, j}^{l} .
$$

The left actions of $e$ and $f$ are twisted derivations in the sense that

$$
\begin{aligned}
& \partial_{e}(\alpha \beta)=\partial_{e}(\alpha) \partial_{k}(\beta)+\partial_{k}^{-1}(\alpha) \partial_{e}(\beta), \\
& \partial_{f}(\alpha \beta)=\partial_{f}(\alpha) \partial_{k}(\beta)+\partial_{k}^{-1}(\alpha) \partial_{f}(\beta)
\end{aligned}
$$

for $\alpha, \beta \in \mathcal{A}$.

Definition 4.2. Define the Hilbert space $\mathscr{H}:=\mathscr{H}_{1} \oplus \mathscr{H}_{-1}$, and represent $\mathscr{B}$ on $\mathscr{H}$ by left multiplication. The Hilbert space $\mathscr{H}$ is graded by $\gamma:=\left(\begin{array}{ll}1 & 0 \\ 0 & -1\end{array}\right)$. Define the weight $\Psi_{R}$ on $\mathscr{B}(\mathcal{H})$ by $\Psi_{R}(T):=\operatorname{Tr}\left(\Delta_{R}^{-\frac{1}{2}} T \Delta_{R}^{-\frac{1}{2}}\right)$. Finally, on a suitable domain in $\mathscr{H}$, define the self-adjoint operator $\mathscr{D}:=\left(\begin{array}{cc}0 & \partial_{e} \\ \partial_{f} & 0\end{array}\right)$.

In fact $(\mathscr{B}, \mathscr{H}, \mathscr{D}, \gamma)$ defines an honest spectral triple, [DS], (i.e., a modular spectral triple with von Neumann algebra $\mathscr{B}(\mathscr{H})$ and weight given by the operator trace) which is $\varepsilon$-summable for all $\varepsilon>0$.

Lemma 4.3. The data $\left(\mathscr{B}, \mathcal{H}, \mathscr{D}, \mathscr{B}(\mathcal{H}), \Psi_{R}\right)$ defines a weakly $\mathrm{Q} C^{\infty}$ even modular spectral triple, which is finitely summable with spectral dimension 2. 
Proof. We first show that the data produces a modular spectral triple. Certainly $\mathcal{B}$ is a separable $*$-subalgebra of $\mathscr{B}(\mathscr{H})$, and by construction the modular automorphism group of $\Psi_{R}$ is $\vartheta_{t}^{-1}$, and $\mathcal{B}$ consists of analytic vectors for $\vartheta_{t}^{-1}$.

Also, the commutators $[\mathcal{D}, \beta]$ extend to bounded operators for all $\beta \in \mathscr{B}$, given by

$$
d \beta:=[\mathcal{D}, \beta]=\left(\begin{array}{cc}
0 & q^{-\frac{1}{2}} \partial_{e}(\beta) \\
q^{\frac{1}{2}} \partial_{f}(\beta) & 0
\end{array}\right) .
$$

We also observe that $\gamma=\gamma^{*}$ and $\gamma^{2}=I$, and by construction $\gamma \mathscr{D}+\mathscr{D} \gamma=0$.

Now, for $T \in \mathscr{B}(\mathscr{H})$ set $T^{+}=(1+\gamma) T(1+\gamma) / 4$ and $T^{-}=(1-\gamma) T(1-\gamma) / 4$. From the definition of the operator trace, and using the normalised Peter-Weyl basis $\xi_{r, j}^{l}:=t_{r, j}^{l} /\left\|t_{r, j}^{l}\right\|$, we find for $T \geq 0$ that

$$
\Psi_{R}(T)=\sum_{l, r} q^{-2 r}\left(\left\langle\xi_{r, 1 / 2}^{l}, T^{+} \xi_{r, 1 / 2}^{l}\right\rangle+\left\langle\xi_{r,-\frac{1}{2}}^{l}, T^{-} \xi_{r,-\frac{1}{2}}^{l}\right\rangle\right) .
$$

We first observe from the above formula that the finite rank operators are in the domain of $\Psi_{R}$, so $\Psi_{R}$ is semi-finite. Next, we see that $\Psi_{R}$ is a sum of vector states with orthogonal support, as the Peter-Weyl basis is orthogonal. Hence $\Psi_{R}$ is strictly semi-finite.

The Peter-Weyl basis elements can be used to construct a common eigenbasis for $\mathscr{D}$ and $\Delta_{R}$ on $\mathscr{H}$, so the spectral projections of $\mathscr{D}$ and $\Delta_{R}$ commute. We conclude that $\mathscr{D}$ is affiliated to the fixed point algebra $\mathcal{M}:=\mathscr{B}(\mathscr{H})^{\vartheta^{-1}}$. All that remains to be proved is that $\left(1+\mathscr{D}^{2}\right)^{-\frac{1}{2}} \in \mathcal{K}\left(\mathcal{M},\left.\Psi_{R}\right|_{\mathcal{M}}\right)$. To establish this, we observe that $\mathscr{D}^{2}$ has the following spectral projections

$$
\mathcal{P}_{l}\left(\begin{array}{c}
t_{r, \frac{1}{2}}^{k} \\
0
\end{array}\right):=\delta_{l, k}\left(\begin{array}{c}
t_{r, \frac{1}{2}}^{k} \\
0
\end{array}\right), \quad \mathcal{P}_{l}\left(\begin{array}{c}
0 \\
t_{r,-\frac{1}{2}}^{k}
\end{array}\right):=\delta_{l, k}\left(\begin{array}{c}
0 \\
t_{r,-\frac{1}{2}}^{k}
\end{array}\right),
$$

for $l=1 / 2,3 / 2, \ldots$, which correspond to the eigenvalues $\left[l+\frac{1}{2}\right]_{q}^{2}$. Now $\Psi_{R}\left(\mathcal{P}_{l}\right)=$ $\sum_{r=-l}^{l} q^{-2 r}=[2 l+1]_{q}$, and the sum $\sum_{l=\frac{1}{2}, \frac{3}{2}, \ldots}\left(1+\left[l+\frac{1}{2}\right]_{q}^{2}\right)^{-\frac{1}{2}}<\infty$ implies that

$$
\left(1+\mathscr{D}^{2}\right)^{-\frac{1}{2}}=\sum_{l=\frac{1}{2}, \frac{3}{2}, \ldots}\left(1+\left[l+\frac{1}{2}\right]_{q}^{2}\right)^{-\frac{1}{2}} \mathcal{P}_{l}
$$

is norm convergent. It follows that $\left(1+\mathscr{D}^{2}\right)^{-\frac{1}{2}} \in \mathcal{K}\left(\mathcal{M},\left.\Psi_{R}\right|_{\mathcal{M}}\right)$ and so $\left(\mathscr{B}, \mathcal{H}, \mathscr{D}, \gamma, \mathscr{B}(\mathscr{H}), \Psi_{R}\right)$ is a modular spectral triple. The spectral dimension is shown to be 2 in [KW].

We now prove that $\mathscr{B} \subset \mathrm{OP}^{0},[\mathscr{D}, \mathscr{B}] \subset \mathrm{w}^{-\mathrm{OP}^{0}}$ so that the modular spectral triple is weakly $\mathrm{Q} C^{\infty}$. The first statement is proved in [NT], Proposition 3.2. To prove the second statement we show that for all $\beta \in \mathscr{B}$ and $z \in \mathbb{C}$, the operators $\left(1+\mathscr{D}^{2}\right)^{-z}[\mathscr{D}, \beta]\left(1+\mathscr{D}^{2}\right)^{z} \in \mathscr{B}(\mathscr{H})$, as per Lemma 3.2. We begin by observing 
that $\mathscr{D}^{2}$ has eigenbasis given by

$$
\mathscr{D}^{2}\left(\begin{array}{c}
t_{r, \frac{1}{2}}^{l} \\
0
\end{array}\right)=\left[l+\frac{1}{2}\right]^{2}\left(\begin{array}{c}
t^{l} \\
r, \frac{1}{2} \\
0
\end{array}\right), \quad \mathscr{D}^{2}\left(\begin{array}{c}
0 \\
t_{r,-\frac{1}{2}}^{l}
\end{array}\right)=\left[l+\frac{1}{2}\right]^{2}\left(\begin{array}{c}
0 \\
t_{r,-\frac{1}{2}}^{l}
\end{array}\right) .
$$

Now we consider $\beta \in \mathscr{B}$ to be of the form $t_{r, 0}^{p}$ (as finite linear combinations of these elements $\operatorname{span} \mathscr{B})$. Then the commutator $\left[\mathcal{D}, t_{r, 0}^{p}\right]=\left(\begin{array}{cc}0 & \kappa_{1} t_{r, 1}^{p} \\ \kappa_{2} t_{r,-1}^{p} & 0\end{array}\right)$ for some coefficients $\kappa_{1}, \kappa_{2}$. We expand the product $t_{r, 0}^{p} t_{s, \frac{1}{2}}^{l}$ using the Clebsch-Gordan coefficients (see [DLSSV], [KS]), giving

$$
\begin{aligned}
\left(1+D^{2}\right)^{-z} t_{r, 0}^{p}\left(1+\mathscr{D}^{2}\right)^{z}\left(\begin{array}{c}
t_{s, \frac{1}{2}}^{l} \\
0
\end{array}\right) & \\
& =\left(1+\left[l+\frac{1}{2}\right]_{q}^{2}\right)^{z} \sum_{k=|l-p|}^{l+p}\left(1+\left[k+\frac{1}{2}\right]_{q}^{2}\right)^{-z} c_{s, r}^{p, l, k}\left(\begin{array}{c}
t_{s+r, \frac{1}{2}}^{k} \\
0
\end{array}\right),
\end{aligned}
$$

where $c_{s, r}^{p, l, k}$ is some product of Clebsch-Gordan coefficients that will be subsumed later.

The norm of $\left(1+\mathscr{D}^{2}\right)^{-z} t_{r, 0}^{p}\left(1+\mathscr{D}^{2}\right)^{z}\left(\begin{array}{c}t_{s, \frac{1}{2}}^{l} \\ 0\end{array}\right)$ can be computed using the orthogonality of the Peter-Weyl basis, so

$$
\begin{aligned}
\left\|\left(1+\mathscr{D}^{2}\right)^{-z} t_{r, 0}^{p}\left(1+\mathscr{D}^{2}\right)^{z}\left(\begin{array}{c}
t^{l} \\
s, \frac{1}{2} \\
0
\end{array}\right)\right\|^{2} \\
\quad=\sum_{k=|l-p|}^{l+p}\left(\frac{1+\left[l+\frac{1}{2}\right]^{2}}{1+\left[k+\frac{1}{2}\right]^{2}}\right)^{2 \operatorname{Re}(z)}\left|c_{s, r}^{p, l, k}\right|^{2}\left\|\left(\begin{array}{c}
t_{s+r, \frac{1}{2}}^{k} \\
0
\end{array}\right)\right\|^{2} .
\end{aligned}
$$

Let $M_{l, p}:=\max _{|l-p| \leq k \leq l+p}\left\{\left(\left(1+\left[l+\frac{1}{2}\right]^{2}\right) /\left(1+\left[k+\frac{1}{2}\right]^{2}\right)\right)^{2 \operatorname{Re}(z)}\right\}$. Then

$$
\begin{aligned}
& \left\|\left(1+\mathscr{D}^{2}\right)^{-z} t_{r, 0}^{p}\left(1+\mathcal{D}^{2}\right)^{z}\left(\begin{array}{c}
t^{l} \\
s, \frac{1}{2} \\
0
\end{array}\right)\right\|^{2} \leq M_{l, p} \sum_{k=|l-p|}^{l+p}\left|c_{s, r}^{p, l, k}\right|^{2}\left\|\left(\begin{array}{c}
t_{s+r, \frac{1}{2}}^{k} \\
0
\end{array}\right)\right\|^{2} \\
& =M_{l, p}\left\|t_{r, 0}^{p}\left(\begin{array}{c}
t_{s, \frac{1}{2}}^{l} \\
0
\end{array}\right)\right\|^{2}
\end{aligned}
$$

It remains to show that there exist finite $M_{p}$ such that $M_{l, p} \leq M_{p}$ for all $l \geq 0$. Let $\varepsilon_{k}=Q\left(1-q^{2 k}\right)$ so that $[k]_{q}=q^{-k} \varepsilon_{k}$. Then for all $l \geq p+\frac{1}{2}$ : if

$$
\frac{1+\left[l+\frac{1}{2}\right]^{2}}{1+\left[|l-p|+\frac{1}{2}\right]^{2}}=\frac{\varepsilon_{l+\frac{1}{2}}^{2}+q^{2 l+1}}{q^{2 p} \varepsilon_{l-p+\frac{1}{2}}^{2}+q^{2 l+1}},
$$


then

$$
\frac{\varepsilon_{1}^{2}}{1+q^{2 p} Q^{2}} \leq \frac{1+\left[l+\frac{1}{2}\right]^{2}}{1+\left[|l-p|+\frac{1}{2}\right]^{2}} \leq \frac{Q^{2}+1}{q^{2 p} \varepsilon_{1}^{2}} .
$$

It follows that the operator $\left(1+\mathscr{D}^{2}\right)^{-1} t_{r, 0}^{p}\left(1+\mathscr{D}^{2}\right)$ is bounded on the set of vectors of the form $\left(\begin{array}{c}t_{s, \frac{1}{2}}^{l} \\ 0\end{array}\right)$. The same calculation can be performed for the vectors $\left(\begin{array}{c}t_{s,-\frac{1}{2}}^{0} \\ { }^{l}\end{array}\right)$, and again for the operators $\left(\begin{array}{cc}0 & t_{r, 1}^{p} \\ 0 & 0\end{array}\right)$ and $\left(\begin{array}{cc}0 & 0 \\ t_{r,-1}^{p} & 0\end{array}\right)$, completing the proof.

4.2. The residue cocycle for the Podleś sphere. Lemma 4.3 shows that the modular spectral triple $(\mathscr{B}, \mathscr{H}, \mathscr{D})$ satisfies the hypotheses of Theorem 3.14. Hence we can employ the resolvent cocycle to compute index pairings with equivariant K-theory, or at least those classes which can be represented as projections over $\mathscr{B}$. As $\Delta_{R}$ implements the modular automorphism $\vartheta$, then it follows that the weight $\Psi_{R}$ is $\vartheta^{-1}$ twisted. The resolvent cocycle, which we denote by $\left(\phi_{m}^{r}\right)_{m=0,2}$, therefore lives in $\vartheta^{-1}$-twisted cohomology.

To simplify the computation of the resolvent cocycle, we would like to have a version of the pseudodifferential calculus. A simple replacement for the pseudodifferential calculus for this example was presented in [NT].

Lemma 4.4 ([NT], Corollary 3.3). Define $\chi:=\left(\begin{array}{cc}q^{-1} & 0 \\ 0 & q\end{array}\right)$ on $\mathscr{H}_{1} \oplus \mathscr{H}_{-1}$. For any $\beta \in \mathscr{B}$ there exists an analytic function $z \mapsto M(z) \in \mathrm{w}_{-} \mathrm{OP}^{0} \subset \mathscr{B}(\mathcal{H})$ with at most linear growth on vertical strips such that

$$
|D|^{-z} d \beta=d \beta \chi^{z}|D|^{-z}+M(z)|D|^{-z-1}=\chi^{-z} d \beta|D|^{-z}+M(z)|D|^{-z-1} .
$$

We can now use this pseudodifferential calculus to simplify the computation of the resolvent cocycle, $\left(\phi_{0}^{r}, \phi_{2}^{r}\right)$, and arrive at a twisted version of the local index formula.

The first simplification we make is to discard the 1 from the resolvent, replacing $R_{s}(\lambda)=\left(\lambda-\left(1+s^{2}+D^{2}\right)\right)^{-1}$ with $R_{s}(\lambda)=\left(\lambda-\left(s^{2}+D^{2}\right)\right)^{-1}$. This is possible because $\mathscr{D}$ is invertible in this example, so we can employ the method of [CPRS4], Section 5.3, in particular [CPRS4], Proposition 5.20. (The transgression cochain defined there is well defined for weakly $\mathrm{Q} C^{\infty}$ modular spectral triples since $\mathscr{D} \in \mathrm{OP}^{1}$, by essentially the same arguments as we employed for the resolvent cocycle). Removing the 1 from the resolvents modifies the resolvent cocycle by coboundaries and cochains holomorphic at $r=-\frac{1}{2}$.

Before proceeding, we recall the detailed summability properties of the spectral triple $(\mathscr{B}, \mathscr{H}, \mathscr{D})$ computed in $[\mathrm{KW}]$.

Lemma 4.5 ([KW], Proposition 1). The function $r \mapsto \operatorname{Tr}\left(\Delta_{R}^{-1} \frac{1}{2}(1 \pm \gamma)|D|^{-3-2 r}\right)$ has a meromorphic continuation to the complex plane which is holomorphic for 
$\operatorname{Re}(r)>-\frac{1}{2}$ and has a simple pole at $r=-\frac{1}{2}$. Furthermore, for all $\beta \in \mathcal{B}$ we have the equality

$$
\operatorname{Res}_{r=-\frac{1}{2}} \operatorname{Tr}\left(\Delta_{R}^{-1} \frac{1}{2}(1 \pm \gamma) \beta|D|^{-3-2 r}\right)=\frac{\left(q^{-1}-q\right)}{2 \ln q^{-1}} \varepsilon(\beta)
$$

where $\varepsilon$ is the counit of $\mathcal{A}$ restricted to $\mathcal{B}$ satisfying $\varepsilon\left(t_{i, 0}^{l}\right)=\delta_{i, 0}$.

The degree zero component $\phi_{0}^{r}$ of the resolvent cocycle is computed from the definition using the Cauchy formula and [CPRS3]. This yields the formula, for $a_{0} \in \mathcal{B}$,

$$
\begin{aligned}
\phi_{0}^{r}\left(a_{0}\right) & =2 \int_{0}^{\infty} \operatorname{Tr}\left(\Delta_{R}^{-1} \gamma \frac{1}{2 \pi i} \int_{l} \lambda^{-1-r} a_{0} R_{s}(\lambda) d \lambda\right) d s \\
& =\frac{\Gamma\left(\frac{1}{2}\right) \Gamma\left(r+\frac{1}{2}\right)}{\Gamma(r+1)} \operatorname{Tr}\left(\Delta_{R}^{-1} \gamma a_{0} \mid D^{-2 r-1}\right) .
\end{aligned}
$$

Since $\operatorname{Tr}\left(\Delta_{R}^{-1} \gamma|\mathcal{D}|^{-2 r-1}\right)=0$ for all sufficiently large $r \in \mathbb{R}$, then taking the trivial continuation to the whole real line gives $\phi_{0}^{r}(I)=0$ for all $r \in \mathbb{R}$. This is the only evaluation of $\phi_{0}^{r}$ needed to compute the index pairing later on.

We can compute $\operatorname{Res}_{r=-\frac{1}{2}} \phi_{0}^{r}$ explicitly, but as the calculation is quite lengthy and we do not require this full computation for computing the index pairing, we just quote the result; see [S] for full details.

The functional $\phi_{0}:=\operatorname{Res}_{r=-\frac{1}{2}} \phi_{0}^{r}$ is supported on the span of the powers $(b c)^{k}$, $k=0,1,2 \ldots$ We have seen that $\phi_{0}(I)=0$. For the remaining values we have

$$
\operatorname{Res}_{r=-\frac{1}{2}} \phi_{0}^{r}(b c)=\frac{1}{2}\left(1-\frac{\gamma}{\ln q^{-1}}\right)-q Q
$$

where $\gamma$ is Euler's constant. For $k=0,1,2, \ldots$ and with $h$ the Haar state, we have

$$
\operatorname{Res}_{r=-\frac{1}{2}} \phi_{0}^{r}\left((b c)^{k+2}\right)=\frac{(-1)^{k+1} q^{k+1}}{1-q^{2 k+2}}=\frac{(-1)^{k+1}}{q^{-k-1}-q^{k+1}}=-\frac{h\left((b c)^{k}\right)}{q^{-1}-q} .
$$

We now compute the degree two term $\phi_{2}^{r}$ of the resolvent cocycle starting with the definition,

$$
\begin{aligned}
& \phi_{2}^{r}\left(a_{0}, a_{1}, a_{2}\right) \\
& \quad=4 \int_{0}^{\infty} s^{2} \operatorname{Tr}\left(\Delta_{R}^{-1} \gamma \frac{1}{2 \pi i} \int_{l} \lambda^{-1-r} a_{0} R_{s}(\lambda) d a_{1} R_{s}(\lambda) d a_{2} R_{s}(\lambda) d \lambda\right) d s .
\end{aligned}
$$

We proceed by employing the pseudodifferential calculus described in Lemma 4.4 in order to rewrite the expression $a_{0} R_{s}(\lambda) d a_{1} R_{s}(\lambda) d a_{2} R_{s}(\lambda)$ by moving all the 
resolvents to the right. From Lemma 4.4, for each $\beta \in \mathscr{B}$ there exist bounded operators $M_{1}, M_{2}$ such that

$$
\begin{aligned}
\left(\lambda-s^{2}-\mathscr{D}^{2}\right) d \beta & =d \beta\left(x \lambda-s^{2}-\chi^{-2} \mathscr{D}^{2}\right)+M_{1}|\mathscr{D}|, \\
\left(\lambda-s^{2}-\chi^{-2} \mathscr{D}^{2}\right) d \beta & =d \beta\left(\lambda-s^{2}-\mathscr{D}^{2}\right)+M_{2}|\mathscr{D}| .
\end{aligned}
$$

This gives the formulae

$$
\begin{aligned}
& R_{S}(\lambda) d \beta=d \beta\left(\lambda-s^{2}-\chi^{-2} \mathscr{D}^{2}\right)^{-1}-R_{S}(\lambda) M_{1}|\mathscr{D}|\left(\lambda-s^{2}-\chi^{-2} \mathscr{D}^{2}\right)^{-1}, \\
& d \beta R_{S}(\lambda)=\left(\lambda-s^{2}-\chi^{-2} \mathscr{D}^{2}\right)^{-1} d \beta+\left(\lambda-s^{2}-\chi^{-2} \mathscr{D}^{2}\right)^{-1} M_{2}|\mathscr{D}| R_{S}(\lambda) .
\end{aligned}
$$

Observe that the operators $R_{s}(\lambda) M_{1}|\mathscr{D}|\left(\lambda-s^{2}-\chi^{-2} \mathscr{D}^{2}\right)^{-1}$ and $\left(\lambda-s^{2}-\right.$ $\left.\chi^{-2} \mathscr{D}^{2}\right)^{-1} M_{2}|\mathscr{D}| R_{s}(\lambda)$ are in w-OP ${ }^{-3}$ by Lemma 4.4. Using this observation, and eq. (4.2), we can move all the resolvents to the right, and in doing so we only introduce errors which are functions holomorphic at $r=-\frac{1}{2}$. More precisely, for any $a_{0}, a_{1}, a_{2} \in \mathscr{B}$, we obtain the formula

$$
\begin{gathered}
\phi_{2}^{r}\left(a_{0}, a_{1}, a_{2}\right)=4 \int_{0}^{\infty} s^{2} \operatorname{Tr}\left(\Delta _ { R } ^ { - 1 } \gamma a _ { 0 } d a _ { 1 } d a _ { 2 } \frac { 1 } { 2 \pi i } \int _ { l } \lambda ^ { - 1 - r } R _ { s } ( \lambda ) \left(\lambda-s^{2}\right.\right. \\
\left.\left.-\chi^{-2} D^{2}\right)^{-1} R_{s}(\lambda) d \lambda\right) d s \\
=4 \int_{0}^{\infty} s^{2} \operatorname{Tr}\left(\Delta _ { R } ^ { - 1 } \gamma a _ { 0 } d a _ { 1 } d a _ { 2 } \frac { 1 } { 2 \pi i } \int _ { l } \lambda ^ { - 1 - r } \left(\lambda-s^{2}\right.\right. \\
\left.\left.-\chi^{-2} D^{2}\right)^{-1} R_{s}(\lambda)^{2} d \lambda\right) d s
\end{gathered}
$$

modulo functions holomorphic at $r=-\frac{1}{2}$. The integral

$$
\int_{l} \lambda^{-1-r}\left(\lambda-s^{2}-\chi^{-2} \mathscr{D}^{2}\right)^{-1} R_{s}(\lambda)^{2} d \lambda
$$

is evaluated on the spectra of the operators $\left(\lambda-s^{2}-\chi^{-2} \mathscr{D}^{2}\right)^{-1}$ and $R_{s}(\lambda)^{2}$. We want to use the Cauchy integral formula, however because there are two poles to consider, $\lambda=s^{2}+\mathscr{D}^{2}$ and $\lambda=s^{2}+\chi^{-2} \mathscr{D}^{2}$, we outline the process.

First note that $\chi$ and $\mathscr{D}^{2}$ are commuting operators with discrete spectra, and they can be simultaneously diagonalised with respect to the direct sum $\mathscr{H}=\mathscr{H}_{1} \oplus \mathscr{H}_{-1}$. Indeed, the integrand in eq. (4.3) has the eigenbasis

$$
\left\{\left(\begin{array}{c}
t_{i, \frac{1}{2}}^{l} \\
0
\end{array}\right),\left(\begin{array}{c}
t_{i,-\frac{1}{2}}^{l} \\
0
\end{array}\right) \mid l-\frac{1}{2} \in \mathbb{N}_{0}, i \in\{-l,-l+1, \ldots, l\}\right\},
$$

on which $\chi^{-2}$ simply acts via multiplication by the scalar $q^{ \pm 2} \neq 1$. We specialise to the eigenbasis in $\mathscr{H}_{1}$, where $\chi^{-2}$ acts via multiplication by $q^{2}$. The argument we now present can be applied analogously to the remaining eigenbasis elements. 
On each eigenvector, the integral in eq. (4.3) reduces to a scalar integral over $\lambda$, where we may apply the usual Cauchy integral formula. The integrand of this scalar integral has two poles; on the eigenbasis elements in $\mathscr{H}_{1}$ described in eq. (4.4) these poles are $\lambda_{1}=s^{2}+q^{2}\left[l+\frac{1}{2}\right]_{q}^{2}$ and $\lambda_{2}=s^{2}+\left[l+\frac{1}{2}\right]_{q}^{2}$, with $\lambda_{1}<\lambda_{2}$. The contour of integration $l$ is a vertical line to the left of the spectrum for all $s \geq 0$.

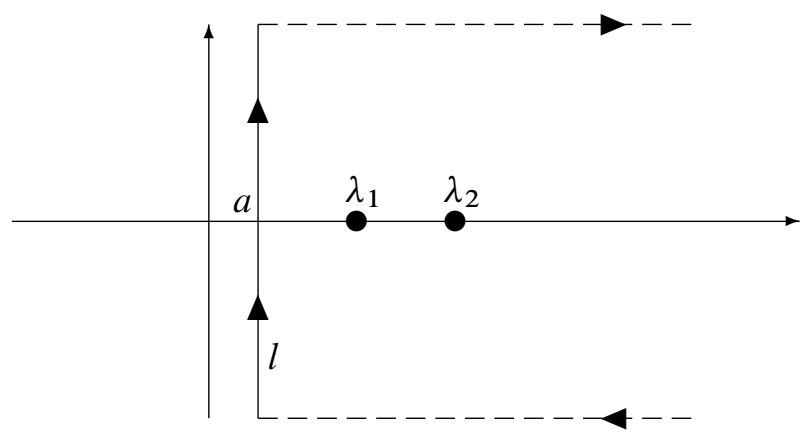

In order to apply the Cauchy integral formula, we modify the contour $l$ by adding a vertical line $l^{\prime}=\left\{a^{\prime}+i v \mid \lambda_{1}<a^{\prime}<\lambda_{2}, v \in \mathbb{R}\right\}$ between the poles $\lambda_{1}$ and $\lambda_{2}$. We integrate along this line in both directions, allowing us to split the integral into two parts.

We denote by $\Gamma_{1}$ the contour obtained by going up along $l$ and down along $l^{\prime}$, and denote by $\Gamma_{2}$ the remaining integration along $l^{\prime}$. Lemma 3.6 shows that the horizontal dashed integrals go to zero.

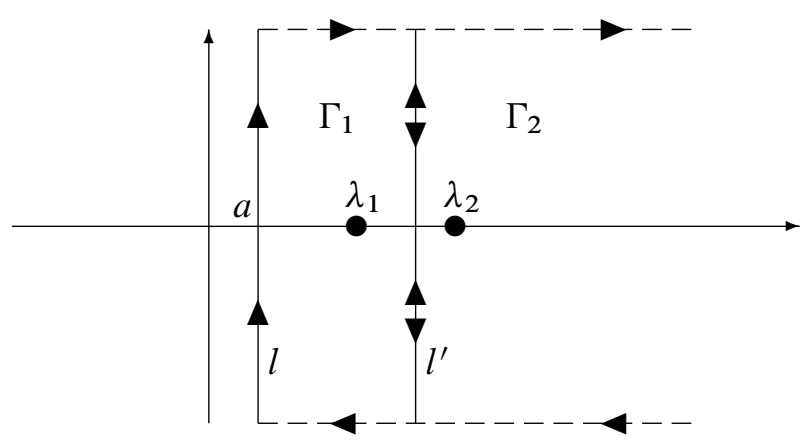

Define

$$
f_{1}(\lambda):=\lambda^{-1-r} R_{s}(\lambda) R_{s}(\lambda), \quad f_{2}(\lambda):=\lambda^{-1-r}\left(\lambda-s^{2}-\chi^{-2} D^{2}\right)^{-1} .
$$

By construction, the function $f_{1}$ is holomorphic on the domain defined by the contour $\Gamma_{1}$, while $f_{2}$ is holomorphic on the domain defined by $\Gamma_{2}$. Therefore, we may apply 
the (scalar) Cauchy integral formula for each contour $\Gamma_{1}$ and $\Gamma_{2}$, so we write

$$
\begin{aligned}
\int_{l} \lambda^{-1-r}\left(\lambda-s^{2}-\chi^{-2} \mathscr{D}^{2}\right)^{-1} R_{s}(\lambda)^{2} d \lambda \\
\quad=\int_{\Gamma_{1}} \frac{f_{1}(\lambda)}{\left(\lambda-s^{2}-\chi^{-2} \mathscr{D}^{2}\right)} d \lambda+\int_{\Gamma_{2}} \frac{f_{2}(\lambda)}{\left(\lambda-s^{2}-\mathscr{D}^{2}\right)^{2}} d \lambda
\end{aligned}
$$

This yields

$$
\begin{aligned}
\frac{1}{2 \pi i} \int_{l} & \lambda^{-1-r}\left(\lambda-s^{2}-\chi^{-2} \mathscr{D}^{2}\right)^{-1} R_{s}(\lambda)^{2} d \lambda \\
= & f_{1}\left(s^{2}+\chi^{-2} \mathscr{D}^{2}\right)+f_{2}^{\prime}\left(s^{2}+\mathscr{D}^{2}\right) \\
= & \left(s^{2}+\chi^{-2} \mathscr{D}^{2}\right)^{-1-r}\left(\chi^{-2}-1\right)^{-2} \mathscr{D}^{-4}-(1+r)\left(s^{2}+\mathfrak{D}^{2}\right)^{-2-r} \\
& \cdot\left(1-\chi^{-2}\right)^{-1} \mathfrak{D}^{-2}-\left(s^{2}+\mathscr{D}^{2}\right)^{-1-r}\left(1-\chi^{-2}\right)^{-2} \mathscr{D}^{-4} .
\end{aligned}
$$

Inserting the result of the Cauchy integral into our previous formula for $\phi_{2}^{r}$ and evaluating the $s$-integrals (see for example [CPRS3], Lemma 5.9) yields

$$
\begin{aligned}
\phi_{2}^{r}\left(a_{0}, a_{1}, a_{2}\right) & \\
= & \frac{\sqrt{\pi} \Gamma\left(r-\frac{1}{2}\right)}{\Gamma(r+1)} \operatorname{Tr}\left(\Delta_{R}^{-1} \gamma a_{0} d a_{1} d a_{2} \chi^{2 r-1}|\mathscr{D}|^{-2 r+1}\left(\chi^{-2}-1\right)^{-2} \mathscr{D}^{-4}\right) \\
& -\frac{\sqrt{\pi} \Gamma\left(r+\frac{1}{2}\right)}{\Gamma(r+1)} \operatorname{Tr}\left(\Delta_{R}^{-1} \gamma a_{0} d a_{1} d a_{2}|\mathscr{D}|^{-2 r-1}\left(1-\chi^{-2}\right)^{-1} \mathscr{D}^{-2}\right) \\
& -\frac{\sqrt{\pi} \Gamma\left(r-\frac{1}{2}\right)}{\Gamma(r+1)} \operatorname{Tr}\left(\Delta_{R}^{-1} \gamma a_{0} d a_{1} d a_{2}|\mathscr{D}|^{-2 r+1}\left(1-\chi^{-2}\right)^{-2} \mathscr{D}^{-4}\right),
\end{aligned}
$$

modulo functions holomorphic at $r=-\frac{1}{2}$. Writing $\Gamma\left(r+\frac{1}{2}\right)=\left(r-\frac{1}{2}\right) \Gamma\left(r-\frac{1}{2}\right)$ and collecting terms, $\phi_{2}^{r}\left(a_{0}, a_{1}, a_{2}\right)$ is given by

$$
\begin{aligned}
\frac{\sqrt{\pi} \Gamma\left(r-\frac{1}{2}\right)}{\Gamma(r+1)} \operatorname{Tr}\left(\Delta_{R}^{-1} \gamma a_{0} d a_{1} d a_{2}|\mathcal{D}|^{-2 r-3}\left(1-\chi^{-2}\right)^{-2}\right. \\
\left.\cdot\left(\chi^{2 r-1}-\left(r-\frac{1}{2}\right)\left(1-\chi^{-2}\right)-1\right)\right) .
\end{aligned}
$$

Observe that

$$
\begin{aligned}
& \Gamma\left(r-\frac{1}{2}\right)\left(\chi^{2 r-1}-\left(r-\frac{1}{2}\right)\left(1-\chi^{-2}\right)-1\right) \\
& \quad=\Gamma\left(r-\frac{1}{2}\right)\left(\chi^{-2}\left(\chi^{2 r+1}-1\right)-\left(r+\frac{1}{2}\right)\left(1-\chi^{-2}\right)\right) \\
& \quad=\left(r+\frac{1}{2}\right) \Gamma\left(r-\frac{1}{2}\right)\left(\chi^{-2}\left(1+\ln \chi^{2}\right)-1\right)+\Gamma\left(r-\frac{1}{2}\right) \chi^{-2} \sum_{n=2}^{\infty} \frac{\left(\ln \chi^{2}\right)^{n}}{n !}\left(r+\frac{1}{2}\right)^{n} .
\end{aligned}
$$


Now $\Gamma\left(r-\frac{1}{2}\right)$ has a simple pole at $r=-\frac{1}{2}$, so the function in eq. (4.5) is holomorphic at $r=-\frac{1}{2}$ with constant term $1-\chi^{-2}\left(1+\ln \chi^{2}\right)$. Therefore

$$
\begin{aligned}
\phi_{2}\left(a_{0}, a_{1}, a_{2}\right): & =\operatorname{Res}_{r=-\frac{1}{2}} \phi_{2}^{r}\left(a_{0}, a_{1}, a_{2}\right) \\
& =\operatorname{Res}_{r=-\frac{1}{2}} \operatorname{Tr}\left(\Delta_{R}^{-1} \gamma a_{0} d a_{1} d a_{2}|D|^{-2 r-3} C\right),
\end{aligned}
$$

where $C=\left(1-\chi^{-2}\left(1+\ln \chi^{2}\right)\right)\left(1-\chi^{-2}\right)^{-2}=\left(\chi^{2}-1-\ln \chi^{2}\right)\left(\chi-\chi^{-1}\right)^{-2}$ is a constant diagonal matrix. Finally, eq. (4.1) yields

$$
a_{0} d a_{1} d a_{2}=\left(\begin{array}{cc}
a_{0} \partial_{e}\left(a_{1}\right) \partial_{f}\left(a_{2}\right) & 0 \\
0 & a_{0} \partial_{f}\left(a_{1}\right) \partial_{e}\left(a_{2}\right)
\end{array}\right),
$$

and so invoking Lemma 4.5 gives the formula

$$
\begin{array}{r}
\phi_{2}\left(a_{0}, a_{1}, a_{2}\right)=\frac{1}{2\left(q^{-1}-q\right) \ln q^{-1}} \\
-\left(q^{-2}-1-\ln q^{-2}\right) \varepsilon\left(a_{0} \partial_{e}\left(a_{1}\right) \partial_{f}\left(a_{2}\right)\right) \\
\left.\left.-1-\ln q^{2}\right) \varepsilon\left(a_{0} \partial_{f}\left(a_{1}\right) \partial_{e}\left(a_{2}\right)\right)\right) .
\end{array}
$$

4.3. Some equivariant projections and their Chern characters. Our aim is to construct representatives in the equivariant K-theory $K_{0}^{\mathbb{T}}(\mathcal{B})$ for the action of the modular automorphism group $\Psi_{R}$, which is given by $\sigma_{t}^{\Psi_{R}}=\vartheta_{t}^{-1}$. These representatives will take the form of projections $p \in M_{N \times N}(\mathcal{B})$ together with a representation $V: \mathbb{T} \rightarrow M_{N \times N}(\mathbb{C})$ such that $p$ is $\vartheta^{-1} \otimes \operatorname{Ad}(V)$-invariant. See [W] for similar constructions.

For $n \in \frac{1}{2} \mathbb{Z}$, define

$$
T_{n}^{l}:=\left(\begin{array}{c}
t_{l, n}^{l} \\
t_{l-1, n}^{l,} \\
\vdots \\
t_{-l, n}^{l}
\end{array}\right) \quad \text { and } \quad P_{n}:=T_{n}^{|n|} T_{n}^{|n| *}
$$

More explicitly,

$$
\begin{aligned}
P_{n} & =\left(\begin{array}{cccc}
t_{|n|, n}^{|n|} t_{|n|, n}^{|n| *} & t_{|n|, n}^{|n|} t_{|n|-1, n}^{|n| *} & \ldots & t_{|n|, n}^{|n|} t_{-|n|, n}^{|n| *} \\
t_{|n|-1, n}^{|n|} t_{|n|, n}^{|n| *} & \ddots & \ddots & \vdots \\
\vdots & \ddots & \ddots & \vdots \\
t_{-|n|, n}^{|n|} t_{|n|, n}^{|n| *} & \ldots & \ldots & t_{-|n|, n}^{|n|} t_{-|n|, n}^{|n| *}
\end{array}\right), \\
\left(P_{n}\right)_{r, s}= & t_{|n|-r+1, n}^{|n|} t_{|n|-s+1, n}^{|n| *} \cdot
\end{aligned}
$$

By construction, $P_{n}^{*}=P_{n}$ and $P_{n} \in M_{(2|n|+1) \times(2|n|+1)}(\mathscr{B})$. Furthermore,

$$
T_{n}^{l *} T_{n}^{l}=\sum_{p=-l}^{l} t_{p, n}^{l *} t_{p, n}^{l}=\operatorname{Id}_{n}
$$


and hence $P_{n}^{2}=P_{n}$, so $P_{n}$ is a projection. Now define $\lambda_{j}=q^{-2 j+2} \in[1, \infty)$ for $j \in\{1,2, \ldots, 2|n|+1\}$ and define $V_{n}: \mathbb{T} \rightarrow M_{2|n|+1}(\mathbb{C})$ by

$$
V_{n, t}:=\left(\begin{array}{cccc}
\lambda_{1}^{i t} & 0 & \ldots & 0 \\
0 & \lambda_{2}^{i t} & \ddots & \vdots \\
\vdots & \ddots & \ddots & 0 \\
0 & \ldots & 0 & \lambda_{2|n|+1}^{i t}
\end{array}\right) .
$$

While we have defined $V_{n}$ to be a real action on $M_{(2|n|+1) \times(2|n|+1)}(\mathbb{C})$, the action is periodic and so induces a circle action. Observe that

$$
\sigma_{t}\left(\left(P_{n}\right)_{r, s}\right)=q^{-2 i t(|n|-r+1-(|n|-s+1))} t_{|n|-r+1, n}^{|n|} t_{|n|-s+1, n}^{|n| *}=q^{2 i t(r-s)}\left(P_{n}\right)_{r, s}
$$

and

$$
\operatorname{Ad}\left(V_{n, t}\right)\left(P_{n}\right)_{r, s}=\left(V_{n, t} P_{n} V_{n, t}^{-1}\right)_{r, s}=\lambda_{r}^{i t}\left(P_{n}\right)_{r, s}\left(\lambda_{s}^{i t}\right)^{-1}=q^{2 i t(s-r)}\left(P_{n}\right)_{r, s .} .
$$

So $P_{n}$ is $\vartheta^{-1} \otimes \operatorname{Ad}\left(V_{n}\right)$-invariant. We define the weight $G: M_{(2|n|+1) \times(2|n|+1)}(\mathbb{C}) \rightarrow$ $\mathbb{C}$ by

$$
G(X):=\operatorname{Tr}\left(V_{n,-i} X\right)
$$

for $X \in M_{(2|n|+1) \times(2|n|+1)}(\mathbb{C})$ and $\left(V_{n,-i}\right)_{k, m}=\delta_{k, m} q^{-2 k+2}$.

We have demonstrated that $P_{n}$ is an equivariant projection for the circle action represented by $V_{n}$, and therefore defines a class in $K_{0}^{\mathbb{T}}(\mathscr{B})$. We now write down the Chern character of this representative, eq. (3.7).

Lemma 4.6. The Chern character of $\left[P_{n}, V_{n}\right]$ is

$$
\begin{aligned}
& \mathrm{Ch}_{0}\left(\left[P_{n}, V_{n}\right]\right)=q^{2(n-|n|)} I, \\
& \mathrm{Ch}_{2}\left(\left[P_{n}, V_{n}\right]\right)=-2 \sum_{k_{0}, k_{1}, k_{2}=0}^{2|n|} q^{-2 k_{0}}\left(t_{|n|-k_{0}, n}^{|n|} t_{|n|-k_{1}, n}^{|n| *}-\frac{1}{2} \delta_{k_{0}, k_{1}}\right) \\
& \otimes t_{|n|-k_{1}, n}^{|n|} t_{|n|-k_{2}, n}^{|n| *} \otimes t_{|n|-k_{2}, n}^{|n|} t_{|n|-k_{0}, n}^{|n| *} .
\end{aligned}
$$

Proof. Using eq. (3.7) we have

$$
\begin{aligned}
\mathrm{Ch}_{0}\left(\left[P_{n}, V_{n}\right]\right) & =\sum_{k_{0}, k_{1}=1}^{2|n|+1}\left(V_{n,-i}\right)_{k_{1}, k_{0}}\left(P_{n}\right)_{k_{0}, k_{1}} \\
& =\left.\sum_{k_{0}, k_{1}=1}^{2|n|+1} \delta_{k_{1}, k_{0}} q^{-2 k_{0}+2} t_{|n|-k_{0}+1, n}^{|n|}\right|_{|n|-k_{1}+1, n} ^{|n| *} .
\end{aligned}
$$

Next we apply the formulae for adjoints, $\left(t_{i, j}^{l}\right)^{*}=(-q)^{j-i} t_{-i,-j}^{l}$ and $t_{i, j}^{l}=$ $(-q)^{j-i}\left(t_{-i,-j}^{l}\right)^{*}$, along with the unitary relations for the Peter-Weyl basis elements, 
[KS], Proposition 16, Chapter 4, to obtain

$$
\mathrm{Ch}_{0}\left(\left[P_{n}, V_{n}\right]\right)=\sum_{k=0}^{2|n|} q^{-2 k} q^{2(n-|n|+k)} t_{k-|n|,-n}^{|n| *} t_{k-|n|,-n}^{|n|}=q^{2(n-|n|)} I .
$$

Finally,

$$
\begin{gathered}
\mathrm{Ch}_{2}\left(\left[P_{n}, V_{n}\right]\right) \\
=-\frac{2 !}{1 !} \sum_{k_{0}, k_{1}, k_{2}, k_{3}=1}^{2|n|+1}\left(V_{n,-i}\right)_{k_{3}, k_{0}}\left(P_{n}-\frac{1}{2}\right)_{k_{0}, k_{1}} \otimes\left(P_{n}\right)_{k_{1}, k_{2}} \otimes\left(P_{n}\right)_{k_{2}, k_{3}} \\
=-2 \sum_{k_{0}, k_{1}, k_{2}=1}^{2|n|+1}\left(V_{n,-i}\right)_{k_{0}, k_{0}}\left(P_{n}-\frac{1}{2}\right)_{k_{0}, k_{1}} \otimes\left(P_{n}\right)_{k_{1}, k_{2}} \otimes\left(P_{n}\right)_{k_{2}, k_{0}} \\
=-2 \sum_{k_{0}, k_{1}, k_{2}=0}^{2|n|} q^{-2 k_{0}}\left(t_{|n|-k_{0}, n}^{|n|} t_{|n|-k_{1}, n}^{|n| *}-\frac{1}{2} \delta_{k_{0}, k_{1}}\right) \\
\otimes t_{|n|-k_{1}, n}^{|n|} t_{|n|-k_{2}, n}^{|n| *} \otimes t_{|n|-k_{2}, n}^{|n|} t_{|n|-k_{0}, n}^{|n| *} .
\end{gathered}
$$

4.4. The index pairing. The resolvent index formula established in Section 3.3 demonstrates that the index pairing defined by the modular spectral triple $\left(\mathscr{B}, \mathscr{H}, \mathscr{D}, \mathscr{B}(\mathscr{H}), \Psi_{R}\right)$ and the equivariant K-theory class defined by the projection $P_{n}$ is given by the formula

$$
\operatorname{Ind}_{\Psi_{R} \otimes G}\left(P_{n}\left(D \otimes \operatorname{Id}_{2|n|+1}\right)^{+} P_{n}\right)=\phi_{2}\left(\mathrm{Ch}_{2}\left(\left[P_{n}, V_{n}\right]\right)\right)+\phi_{0}\left(\mathrm{Ch}_{0}\left(\left[P_{n}, V_{n}\right]\right)\right) .
$$

Now that we have explicit formulae for the cocycle $\left(\phi_{0}, \phi_{2}\right)$ and the cycle $\mathrm{Ch}_{*}\left(\left[P_{n}, V_{n}\right]\right)$, the computation is straightforward.

Proposition 4.7. The evaluation of $\phi_{2}$ on $\mathrm{Ch}_{2}\left(\left[P_{n}, V_{n}\right]\right)$ is

$$
\phi_{2}\left(\mathrm{Ch}_{2}\left(\left[P_{n}, V_{n}\right]\right)\right)=q^{-2|n|}[2 n]_{q} .
$$

Proof. Recalling the formula for $\phi_{2}$ from eq. (4.6) and from Lemma 4.6 the expression for $\mathrm{Ch}_{2}\left(\left[P_{n}, V_{n}\right]\right)$, we compute

$$
\begin{aligned}
& \varepsilon\left(\left(t_{|n|-k_{0}, n}^{|n|} t_{|n|-k_{1}, n}^{|n| *}-\frac{1}{2} \delta_{k_{0}, k_{1}}\right) \partial_{e}\left(\left.t_{|n|-k_{1}, n}^{|n|}\right|_{|n|-k_{2}, n} ^{|n| *}\right) \partial_{f}\left(t_{|n|-k_{2}, n}^{|n|} t_{|n|-k_{0}, n}^{|n| *}\right)\right) \\
& \quad=\left(\delta_{|n|-k_{0}, n} \delta_{|n|-k_{1}, n}-\frac{1}{2} \delta_{k_{0}, k_{1}}\right) \varepsilon\left(\partial_{e}\left(t_{|n|-k_{1}, n}^{|n|} t_{|n|-k_{2}, n}^{|n| *}\right) \varepsilon\left(\partial_{f}\left(t_{|n|-k_{2}, n}^{|n|} t_{|n|-k_{0}, n}^{|n| *}\right)\right)\right. \\
& \quad=\delta_{k_{0}, k_{1}}\left(\delta_{k_{0},|n|-n}-\frac{1}{2}\right) \varepsilon\left(\partial_{e}\left(t_{|n|-k_{1}, n}^{|n|} t_{|n|-k_{2}, n}^{|n| *}\right) \varepsilon\left(\partial_{f}\left(t_{|n|-k_{2}, n}^{|n|} t_{|n|-k_{1}, n}^{|n| *}\right)\right) .\right.
\end{aligned}
$$

We observe that this expression is zero for the case $n=0$, because $\partial_{e}(I)=$ $\partial_{f}(I)=0$. So for the remainder we consider only nonzero $n$. Observe that $t_{|n|-k_{2}, n}^{|n|} t_{|n|-k_{1}, n}^{|n| *}=\left(t_{|n|-k_{1}, n}^{|n|} t_{|n|-k_{2}, n}^{|n| *}\right)^{*}$. Now we use the property that $(g \triangleright \alpha)^{*}=$ 
$S(g)^{*} \triangleright \alpha^{*}$ for all $g \in \mathcal{U}_{q}\left(s u_{2}\right)$ and $\alpha \in \mathcal{A}$, so that $\varepsilon\left(\partial_{e}\left(\alpha^{*}\right)\right)=-q \varepsilon\left(\partial_{f}(\alpha)^{*}\right)$ and $\varepsilon\left(\partial_{f}\left(\alpha^{*}\right)\right)=-q^{-1} \varepsilon\left(\partial_{e}(\alpha)^{*}\right)$. Then

$$
\begin{gathered}
\varepsilon\left(\left(t_{|n|-k_{0}, n}^{|n|} t_{|n|-k_{1}, n}^{|n| *}-\frac{1}{2} \delta_{k_{0}, k_{1}}\right) \partial_{e}\left(t_{|n|-k_{1}, n}^{|n|} t_{|n|-k_{2}, n}^{|n| *}\right) \partial_{f}\left(t_{|n|-k_{2}, n}^{|n|} t_{|n|-k_{0}, n}^{|n| *}\right)\right) \\
=-q^{-1} \delta_{k_{0}, k_{1}}\left(\delta_{k_{0},|n|-n}-\frac{1}{2}\right) \varepsilon\left(\partial_{e}\left(t_{|n|-k_{1}, n}^{|n|} t_{|n|-k_{2}, n}^{|n| *}\right)\right)^{2},
\end{gathered}
$$

and similarly

$$
\begin{gathered}
\varepsilon\left(\left(t_{|n|-k_{0}, n}^{|n|} t_{|n|-k_{1}, n}^{|n| *}-\frac{1}{2} \delta_{k_{0}, k_{1}}\right) \partial_{f}\left(t_{|n|-k_{1}, n}^{|n|} t_{|n|-k_{2}, n}^{|n| *}\right) \partial_{e}\left(t_{|n|-k_{2}, n}^{|n|} t_{|n|-k_{0}, n}^{|n| *}\right)\right) \\
=-q^{-1} \delta_{k_{0}, k_{1}}\left(\delta_{k_{0},|n|-n}-\frac{1}{2}\right) \varepsilon\left(\partial_{e}\left(t_{|n|-k_{2}, n}^{|n|} t_{|n|-k_{1}, n}^{n \mid *}\right)\right)^{2} .
\end{gathered}
$$

Using the twisted derivation property of $\partial_{e}$ on $\mathcal{A}$, we find, for $r, s \in\{0, \ldots, 2|n|\}$,

$$
\begin{aligned}
\varepsilon\left(\partial_{e}(\right. & \left.\left.t_{|n|-r, n}^{|n|} t_{|n|-s, n}^{|n| *}\right)\right)^{2} \\
& =\varepsilon\left(\partial_{e}\left(t_{|n|-r, n}^{|n|}\right) \partial_{k}\left(t_{|n|-s, n}^{|n| *}\right)+\partial_{k}^{-1}\left(t_{|n|-r, n}^{|n|}\right) \partial_{e}\left(t_{|n|-s, n}^{|n| *}\right)\right)^{2} \\
& =q^{-2 n}\left(\varepsilon\left(\partial_{e}\left(t_{|n|-r, n}^{|n|}\right)\right) \delta_{s,|n|-n}+\delta_{r,|n|-n} \varepsilon\left(\partial_{e}\left(t_{|n|-s, n}^{|n| *}\right)\right)\right)^{2} \\
& =q^{-2 n}\left(\varepsilon\left(\partial_{e}\left(t_{|n|-r, n}^{|n|}\right)\right) \delta_{s,|n|-n}-q \delta_{r,|n|-n} \varepsilon\left(\partial_{f}\left(t_{|n|-s, n}^{|n|}\right)\right)\right)^{2} \\
& =q^{-2 n}\left(\varepsilon\left(\kappa_{n+1}^{|n|} t_{|n|-r, n+1}^{|n|}\right) \delta_{s,|n|-n}-q \delta_{r,|n|-n} \varepsilon\left(\kappa_{n}^{|n|} t_{|n|-s, n-1}^{|n|}\right)\right)^{2} \\
& =q^{-2 n}\left(\kappa_{n+1}^{|n|} \delta_{r,|n|-n-1} \delta_{s,|n|-n}-q \kappa_{n}^{|n|} \delta_{r,|n|-n} \delta_{s,|n|-n+1}\right)^{2} \\
& =q^{-2 n}\left(\left(\kappa_{n+1}^{|n|}\right)^{2} \delta_{r,|n|-n-1} \delta_{s,|n|-n}+q^{2}\left(\kappa_{n}^{|n|}\right)^{2} \delta_{r,|n|-n} \delta_{s,|n|-n+1}\right),
\end{aligned}
$$

where $\kappa_{j}^{l}=\left([l+j]_{q}[l-j+1]_{q}\right)^{\frac{1}{2}}$. Combining these results with the formula for $\operatorname{Res}_{r=-\frac{1}{2}} \phi_{2}^{r}$ and the expression for $\mathrm{Ch}_{2}\left(\left[P_{n}, V\right]\right)$ gives

$$
\begin{aligned}
\phi_{2}\left(\mathrm{Ch}_{2}(\right. & {\left.\left.\left[P_{n}, V_{n}\right]\right)\right) } \\
= & \frac{-2}{2\left(q^{-1}-q\right) \ln q^{-1}} \sum_{k_{0}, k_{1}, k_{2}=0}^{2|n|} q^{-2 k_{0}}\left(-q^{-1} \delta_{k_{0}, k_{1}}\left(\delta_{k_{0},|n|-n}-\frac{1}{2}\right)\right) \\
& \cdot\left(\left(q^{-2}-1-\ln q^{-2}\right) \varepsilon\left(\partial_{e}\left(t_{|n|-k_{1}, n}^{|n|} t_{|n|-k_{2}, n}^{|n| *}\right)\right)^{2}\right. \\
& \left.-\left(q^{2}-1-\ln q^{2}\right) \varepsilon\left(\partial_{e}\left(t_{|n|-k_{2}, n}^{|n|} t_{|n|-k_{1}, n}^{|n| *}\right)\right)^{2}\right) \\
= & \frac{q^{-1}}{\left(q^{-1}-q\right) \ln q^{-1}} \sum_{k_{1}, k_{2}=0}^{2|n|} q^{-2 k_{1}}\left(\delta_{k_{1},|n|-n}-\frac{1}{2}\right) q^{-2 n} \\
& \cdot\left(( q ^ { - 2 } - 1 - \operatorname { l n } q ^ { - 2 } ) \left(\left(\kappa_{n+1}^{|n|}\right)^{2} \delta_{k_{1},|n|-n-1} \delta_{k_{2},|n|-n}\right.\right. \\
& \left.+q^{2}\left(\kappa_{n}^{|n|}\right)^{2} \delta_{k_{1},|n|-n} \delta_{k_{2},|n|-n+1}\right) \\
& -\left(q^{2}-1-\ln q^{2}\right)\left(\left(\kappa_{n+1}^{|n|}\right)^{2} \delta_{k_{2},|n|-n-1} \delta_{k_{1},|n|-n}\right. \\
& \left.\left.+q^{2}\left(\kappa_{n}^{|n|}\right)^{2} \delta_{k_{2},|n|-n} \delta_{k_{1},|n|-n+1}\right)\right) .
\end{aligned}
$$


The resolvent cocycle in twisted cyclic cohomology and a local index formula

Using $\left(\delta_{k_{1},|n|-n}-\frac{1}{2}\right) \delta_{k_{1},|n|-n}=\frac{1}{2} \delta_{k_{1},|n|-n}$ and $\left(\delta_{k_{1},|n|-n}-\frac{1}{2}\right) \delta_{k_{1},|n|-n \pm 1}=$ $-\frac{1}{2} \delta_{k_{1},|n|-n \pm 1}$ yields

$$
\begin{aligned}
\operatorname{Res}_{r=-} & \phi_{2}^{r}\left(\operatorname{Ch}_{2}\left(\left[P_{n}, V_{n}\right]\right)\right) \\
= & \frac{q^{-1}}{\left(q^{-1}-q\right) \ln q^{-1}} \sum_{k_{1}, k_{2}=0}^{2|n|} \frac{1}{2} q^{-2 k_{1}-2 n}\left(( q ^ { - 2 } - 1 - \operatorname { l n } q ^ { - 2 } ) \left(-\left(\kappa_{n+1}^{|n|}\right)^{2}\right.\right. \\
& \left.\cdot \delta_{k_{1},|n|-n-1} \delta_{k_{2},|n|-n}+q^{2}\left(\kappa_{n}^{|n|}\right)^{2} \delta_{k_{1},|n|-n} \delta_{k_{2},|n|-n+1}\right) \\
& -\left(q^{2}-1-\ln q^{2}\right)\left(\left(\kappa_{n+1}^{|n|}\right)^{2} \delta_{k_{1},|n|-n} \delta_{k_{2},|n|-n-1}\right. \\
& \left.\left.-q^{2}\left(\kappa_{n}^{|n|}\right)^{2} \delta_{k_{1},|n|-n+1} \delta_{k_{2},|n|-n}\right)\right) .
\end{aligned}
$$

We can reduce the different summations over $k_{1}$ and $k_{2}$ down to two distinct sums, either

$$
\sum_{k=0}^{2|n|} \delta_{k,|n|-n-1}=\delta_{n,-|n|} \quad \text { or } \quad \sum_{k=0}^{2|n|} \delta_{k,|n|-n+1}=\delta_{n,|n|} .
$$

Hence

$$
\begin{aligned}
& \phi_{2}\left(\mathrm{Ch}_{2}\left(\left[P_{n}, V_{n}\right]\right)\right) \\
&=\frac{q^{-1}}{2\left(q^{-1}-q\right) \ln q^{-1}}\left(( q ^ { - 2 } - 1 - \operatorname { l n } q ^ { - 2 } ) \left(-\left(\kappa_{n+1}^{|n|}\right)^{2} \delta_{n,-|n|} q^{-2(|n|-n-1)-2 n}\right.\right. \\
&\left.+q^{2}\left(\kappa_{n}^{|n|}\right)^{2} \delta_{n,|n|} q^{-2(|n|-n)-2 n}\right)-\left(q^{2}-1-\ln q^{2}\right)\left(\left(\kappa_{n+1}^{|n|}\right)^{2} \delta_{n,-|n|}\right. \\
&\left.\left.\cdot q^{-2(|n|-n)-2 n}-q^{2}\left(\kappa_{n}^{|n|}\right)^{2} \delta_{n,|n|} q^{-2(|n|-n+1)-2 n}\right)\right) \\
&= \frac{q^{-1}}{2\left(q^{-1}-q\right) \ln q^{-1}}\left(( q ^ { - 2 } - 1 - \operatorname { l n } q ^ { - 2 } ) \left(-\left(\kappa_{1-|n|}^{|n|}\right)^{2} \delta_{n,-|n|} q^{-2|n|+2}\right.\right. \\
&\left.+q^{2}\left(\kappa_{|n|}^{|n|}\right)^{2} \delta_{n,|n|} q^{-2|n|}\right)-\left(q^{2}-1-\ln q^{2}\right)\left(\left(\kappa_{1-|n|}^{|n|}\right)^{2} \delta_{n,-|n|} q^{-2|n|}\right. \\
&\left.\left.-q^{2}\left(\kappa_{|n|}^{|n|}\right)^{2} \delta_{n,|n|} q^{-2|n|-2}\right)\right) .
\end{aligned}
$$

Observe that $\left(\kappa_{1-|n|}^{|n|}\right)^{2}=\left(\kappa_{|n|}^{|n|}\right)^{2}=[2|n|]_{q}$ as $[1]_{q}=1$, and so $\phi_{2}\left(\mathrm{Ch}_{2}\left(\left[P_{n}, V_{n}\right]\right)\right)$

$$
\begin{aligned}
= & \frac{q^{-1}}{2\left(q^{-1}-q\right) \ln q^{-1}}[2|n|]_{q} q^{-2|n|} \\
& \cdot\left(\left(q^{-2}-1-\ln q^{-2}\right) q^{2}\left(\delta_{n,|n|}-\delta_{n,-|n|}\right)-\left(q^{2}-1-\ln q^{2}\right)\left(\delta_{n,-|n|}-\delta_{n,|n|}\right)\right) \\
= & \frac{q^{-2|n|-1}[2|n|]_{q}}{2\left(q^{-1}-q\right) \ln q^{-1}}\left(\delta_{n,|n|}-\delta_{n,-|n|}\right)\left(\left(q^{-2}-1-\ln q^{-2}\right) q^{2}+\left(q^{2}-1-\ln q^{2}\right)\right) \\
= & \frac{q^{-2|n|-1}[2|n|]_{q}}{2\left(q^{-1}-q\right) \ln q^{-1}}\left(\delta_{n,|n|}-\delta_{n,-|n|}\right)\left(-q^{2} \ln q^{-2}-\ln q^{2}\right)
\end{aligned}
$$




$$
\begin{aligned}
& =\frac{q^{-2|n|-1}[2|n|]_{q}}{2\left(q^{-1}-q\right) \ln q^{-1}}\left(\delta_{n,|n|}-\delta_{n,-|n|}\right)\left(1-q^{2}\right) \ln q^{-2} \\
& =q^{-2|n|}[2|n|]_{q}\left(\delta_{n,|n|}-\delta_{n,-|n|}\right) .
\end{aligned}
$$

Considering $n \neq 0$, then $\left(\delta_{n,|n|}-\delta_{n,-|n|}\right)=\operatorname{sgn}(n)$ and $\operatorname{sgn}(n)[2|n|]_{q}=[2 n]_{q}$. As $[0]_{q}=0$, then for all $n \in \frac{1}{2} \mathbb{Z}$ we have

$$
\phi_{2}\left(\mathrm{Ch}_{2}\left(\left[P_{n}, V_{n}\right]\right)\right)=q^{-2|n|}[2 n]_{q}
$$

We can now write down the index pairing and compute the classical limit as $q \rightarrow 1$.

Theorem 4.8. For $N \in \mathbb{Z}$, the index pairing of the modular spectral triple $\left(\mathscr{B}, \mathscr{H}, \mathcal{D}, \mathcal{B}(\mathscr{H}), \Psi_{R}\right)$ with the equivariant projections $P_{N / 2}$ is

$$
\operatorname{Ind}\left(P_{N / 2}\left(\mathscr{D} \otimes \operatorname{Id}_{|N|+1}\right)^{+} P_{N / 2}\right)=q^{-|N|}[N]_{q} .
$$

The classical limit of the index as $q \rightarrow 1$ is

$$
\lim _{q \rightarrow 1} \operatorname{Ind}\left(P_{N / 2}\left(\mathscr{D} \otimes \operatorname{Id}_{|N|+1}\right)^{+} P_{N / 2}\right)=N .
$$

Proof. First, the degree zero contribution is $\phi_{0}\left(\mathrm{Ch}_{0}\left(\left[P_{N / 2}, V_{N / 2}\right]\right)\right)=0$. This follows from $\phi_{0}(I)=0$, and from Lemma 4.6, which gives $\mathrm{Ch}_{0}\left(\left[P_{N / 2}, V_{N / 2}\right]\right)=$ $q^{(N-|N|)} I$. Thus the index pairing is computed just with the degree 2 part, which comes from Proposition 4.7. To compute the classical limit of the index we recall that $\lim _{q \rightarrow 1}[N]_{q}=N$ (see for example $[\mathrm{KS}]$ ).

We conclude with some comments on how the index depends on the representation

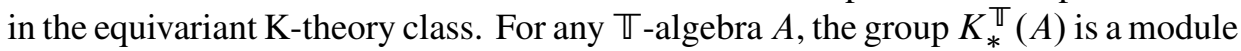
over the group ring $\mathbb{Z}[\mathbb{T}$. This allows us to hold the projection or unitary constant, but vary the representation. This does change the equivariant K-theory class, and this can be detected by the index pairing.

In particular, if the $V_{n}$ used here is rescaled by $q^{2|n| i t}$ to obtain a new representation $V_{n}^{\prime}$, then the index is likewise rescaled by $q^{2|n|}$, and we obtain an index of $[2 n]_{q}$. This observation helps in comparing our results with other authors' results, particularly Wagner's, [W]. We thank the referee for pointing this out to us.

\section{References}

[BeF] M.-T. Benameur and T. Fack, Type II non-commutative geometry. I. Dixmier trace in von Neumann algebras. Adv. Math. 199 (2006), 29-87. Zbl 1092.46050 MR 2186918 
[B] B. Blackadar, K-theory for operator algebras. 2nd ed., Math. Sci. Res. Inst. Publ. 5, Cambridge University Press, Cambridge 1998. Zbl 0913.46054 MR 1656031

[BR] O. Bratteli and D. W. Robinson, Operator algebras and quantum statistical mechanics 2. 2nd ed., Texts Monogr. Phys, Springer-Verlag, Berlin 1997. Zbl 0903.46066 MR 1441540

[CGRS2] A. L. Carey, V. Gayral, A. Rennie, and F. Sukochev, Index theory for locally compact noncommutative spaces. Mem. Amer. Math. Soc., to appear. arXiv:1107.0805

[CMR] A. Carey, M. Marcolli, and A. Rennie, Modular index invariants of Mumford curves. In Noncommutative geometry, arithmetic, and related topics, Johns Hopkins Univ. Press, Baltimore, MD 2011, 31-73. Zbl 1270.46065 MR 2907004

[CNNR] A. L. Carey, S. Neshveyev, R. Nest, and A. Rennie, Twisted cyclic theory, equivariant $K K$-theory and KMS states. J. Reine Angew. Math. 650 (2011), 161-191. Zbl 1218.19002 MR 2770560

[CP1] A. Carey and J. Phillips, Unbounded Fredholm modules and spectral flow. Canad. J. Math. 50 (1998), 673-718. Zbl 0915.46063 MR 1638603

[CP2] A. Carey and J. Phillips, Spectral flow in Fredholm modules, eta invariants and the JLO cocycle. K-Theory 31 (2004), 135-194. Zbl 1051.19004 MR 2053481

[CPPR] A. L. Carey, J. Phillips, I. F. Putnam, and A. Rennie, Families of type III KMS states on a class of $C^{*}$-algebras containing $O_{n}$ and $Q_{\mathbb{N}}$. J. Funct. Anal. 260 (2011), 1637-1681. Zbl 1217.46039 MR 2754888

[CPR2] A. L. Carey, J. Phillips, and A. Rennie, Twisted cyclic theory and an index theory for the gauge invariant KMS state on the Cuntz algebra $O_{n}$. J. K-Theory 6 (2010), 339-380. Zbl 1220.19004 MR 2735089

[CPRS2] A. L. Carey, J. Phillips, A. Rennie, and F. A. Sukochev, The local index formula in semifinite von Neumann algebras I: Spectral flow. Adv. Math. 202 (2006), 451-516. Zbl 1118.46060 MR 2222358

[CPRS3] A. L. Carey, J. Phillips, A. Rennie, and F. A. Sukochev, The local index formula in semifinite von Neumann algebras II: The even case. Adv. Math. 202 (2006), 517-554. Zbl 1101.46045 MR 2222359

[CPRS4] A. L. Carey, J. Phillips, A. Rennie, and F. A. Sukochev, The Chern character of semifinite spectral triples. J. Noncommut. Geom. 2 (2008), 141-193. Zbl 1157.46045 MR 2383362

[CRT] A. L. Carey, A. Rennie, and K. Tong, Spectral flow invariants and twisted cyclic theory for the Haar state on $\mathrm{SU}_{q}$ (2). J. Geom. Phys. 59 (2009), 1431-1452. Zbl 1175.46064 MR 2560289

[C] A. Connes, Noncommutative geometry. Academic Press, San Diego, CA, 1994. Zbl 0818.46076 MR 1303779

[CM] A. Connes and H. Moscovici, The local index formula in noncommutative geometry. Geom. Funct. Anal. 5 (1995), 174-243. Zbl 0960.46048 MR 1334867

[DLSSV] L. Dạbrowski, G. Landi, A. Sitarz, W. van Suijlekom, and J. C. Várilly, The Dirac operator on $S U_{q}$ (2). Comm. Math. Phys. 259 (2005), 729-759. Zbl 1090.58504 MR 2174423 
[DS] L. Dạbrowski and A. Sitarz, Dirac operator on the standard Podleś quantum sphere. In Noncommutative geometry and quantum groups (Warsaw, 2001), Banach Center Publ. 61, Polish Acad. Sci., Warsaw 2003, 49-58. Zbl 1061.58004 MR 2024421

[GVF] J. M. Gracia-Bondía, J. C. Várilly, and H. Figueroa, Elements of noncommutative geometry. Birkhäuser Adv. Texts, Birkhäuser, Boston 2001. Zbl 0958.46039 MR 1789831

[H] T. Hadfield, Twisted cyclic homology of all Podleś quantum spheres. J. Geom. Phys. 57 (2007), 339-351. Zbl 1119.58004 MR 2271192

[HK] T. Hadfield and U. Krähmer, Twisted homology of quantum SL(2) - Part II. $J$. K-Theory 6 (2010), 69-98. Zbl 1201.58003 MR 2672153

[Hig] N. Higson, The local index formula in noncommutative geometry. In Contemporary developments in algebraic $K$-theory, ICTP Lect. Notes 15, Abdus Salam Int. Cent. Theoret. Phys., Trieste 2004, 443-536. Zbl 1122.58015 MR 2175637 http://publications.ictp.it/lns/vol15.html

[KNR] J. Kaad, R. Nest, and A. Rennie, $K K$-theory and spectral flow in von Neumann algebras. J. K-Theory 10 (2012), 241-277. Zbl 1260.19001 MR 3004169

[K] G. G. Kasparov, The operator $K$-functor and extensions of $C^{*}$-algebras. Izv. Akad. Nauk SSSR Ser. Mat. 44 (1980), 571-636; English transl. Math. USSR-Izv. 16(1981), 513-572. Zbl 0464.46054 MR 582160

[KS] A. Klimyk and K. Schmüdgen, Quantum groups and their representations. Texts Monogr. Phys., Springer-Verlag, Berlin 1997. Zbl 0891.17010 MR 1492989

[KW] U. Krähmer and E. Wagner, A residue formula for the fundamental Hochschild class on the Podleś sphere. J. K-Theory 12 (2013), 257-271. Zbl 06251682 MR 3142364

[Kuc] D. Kucerovsky, The $K K$-product of unbounded modules. K-Theory 11 (1997), 17-34. Zbl 0871.19004 MR 1435704

[KMT] J. Kustermans, G. J. Murphy, and L. Tuset, Differential calculi over quantum groups and twisted cyclic cocycles. J. Geom. Phys. 44 (2003), 570-594. Zbl 1029.46114 MR 1943179

[NT] S. Neshveyev and L. Tuset, A local index formula for the quantum sphere. Comm. Math. Phys. 254 (2005), 323-341. Zbl 1079.58021 MR 2117628

[Ph] J. Phillips, Self-adjoint Fredholm operators and spectral flow. Canad. Math. Bull. 39 (1996), 460-467. Zbl 0878.19001 MR 1426691

[SW] K. Schmüdgen and E. Wagner, Dirac operator and a twisted cyclic cocycle on the standard Podleś quantum sphere. J. Reine Angew. Math. 574 (2004), 219-235. Zbl 1067.46070 MR 2099116

[S] R. Senior, Modular spectral triples and KMS states in noncommutative geometry. $\mathrm{PhD}$ thesis, Australian National University, Canberra 2011.

[T] M. Takesaki, Theory of operator algebras II. Encyclopaedia Math. Sci. 125, Springer-Verlag, Berlin 2003. Zbl 1059.46031 MR 1943006

[W] E. Wagner, On the noncommutative Spin geometry of the standard Podles sphere and index computations. J. Geom. Phys. 59 (2009), 998-1016. Zbl 1182.58005 MR 2536858 
Received November 25, 2011

A. Rennie, School of Mathematics and Applied Statistics, University of Wollongong, NSW 2522, Australia

E-mail: renniea@uow.edu.au

R. Senior, Mathematical Sciences Institute, Australian National University, Canberra, ACT 0200, Australia

E-mail: roger.senior@anu.edu.au 